\title{
ON THE RESTRICTED CONFORMAL GROUP OF THE $(1+n)$-EINSTEIN STATIC UNIVERSE
}

\author{
OLIMJON ESHKOBILOV, EMILIO MUSSO, AND LORENZO NICOLODI
}

\begin{abstract}
Explicit models for the restricted conformal group of the Einstein static universe of dimension greater than two and for its universal covering group are constructed. Based on these models, as an application we determine all oriented and time-oriented conformal Lorentz manifolds whose restricted conformal group has maximal dimension. They amount to the Einstein static universe itself and two countably infinite series of its compact quotients.
\end{abstract}

\section{INTRODUCTION}

Let $\mathcal{E}_{\mathrm{I}}^{1, n}$ and $\mathcal{E}_{\mathrm{II}}^{1, n}$ denote the conformal compactifications of Minkowski $(n+1)$ space $(n \geq 2)$, realized respectively as the sets of oriented and unoriented null lines through the origin in pseudo-Euclidean space $\mathbb{R}^{2, n+1}$. Endowed with the Lorentz structures inherited from $\mathbb{R}^{2, n+1}$, they are known in the literature with the name of (compact) $(1+n)$-Einstein universes. Topologically, $\mathcal{E}_{\mathrm{I}}^{1, n} \cong \mathbb{S}^{1} \times \mathbb{S}^{n}$ and $\mathcal{E}_{\mathrm{II}}^{1, n} \cong \mathbb{S}^{1} \times \mathbb{S}^{n} /\{ \pm 1\}$, where -1 acts by the antipodal map on both factors. The space $\mathcal{E}_{\mathrm{I}}^{1, n}$ is orientable. Instead, if $n$ is even, $\mathcal{E}_{\mathrm{II}}^{1, n}$ is not orientable, and $\mathcal{E}_{\mathrm{I}}^{1, n}$ is an orientable double covering of $\mathcal{E}_{\mathrm{II}}^{1, n}$. The $(1+n)$-Einstein static universe is the product space $\mathcal{E}^{1, n} \cong \mathbb{R} \times \mathbb{S}^{n}$ with the Lorentz product metric $-d t^{2}+g_{\mathbb{S}^{n}}$, where $g_{\mathbb{S}^{n}}$ denotes the standard metric of $\mathbb{S}^{n}$ (cf. [6, 15, 16]). The space $\mathcal{E}^{1, n}$ is the universal covering of both $\mathcal{E}_{\mathrm{I}}^{1, n}$ and $\mathcal{E}_{\mathrm{II}}^{1, n}$.

The Einstein universes $\mathcal{E}_{\mathrm{I}}^{1, n}, \mathcal{E}_{\mathrm{II}}^{1, n}$ ( $n$ odd), and $\mathcal{E}^{1, n}$ are examples of oriented, time-oriented, conformal Lorentz manifolds of dimension $n+1$. For an oriented, time-oriented, conformal Lorentz manifold $(\mathbb{M},[g])$ of dimension $n+1 \geq 3$, we let $\mathcal{C}_{+}^{\uparrow}(\mathbb{M})$ denote the restricted conformal group of $\mathbb{M}$, i.e., the group of conformal transformations preserving orientation and time-orientation. It is well-known that $\mathcal{C}_{+}^{\uparrow}\left(\mathcal{E}_{\mathrm{I}}^{1, n}\right) \cong \mathrm{O}_{+}^{\uparrow}(2, n+1)$ and $\mathcal{C}_{+}^{\uparrow}\left(\mathcal{E}_{\mathrm{II}}^{1, n}\right) \cong \mathrm{O}_{+}^{\uparrow}(2, n+1) /\{ \pm I\}$, where $\mathrm{O}_{+}^{\uparrow}(2, n+1)$ is the identity component of $\mathrm{O}(2, n+1)$, the pseudo-orthogonal group of a scalar product of signature $(2, n+1)$ (cf. [9, 12] and Section 2.6). As for $\mathcal{E}^{1, n}$, it is known that $\mathcal{C}_{+}^{\uparrow}\left(\mathcal{E}^{1, n}\right) \cong \widehat{\mathrm{O}}_{+}^{\uparrow}(2, n+1)$, where $\widehat{\mathrm{O}}_{+}^{\uparrow}(2, n+1)$ is a central extension of $\mathrm{O}_{+}^{\uparrow}(2, n+1)$ which can be obtained as a quotient of index 2 of the universal

2000 Mathematics Subject Classification. 53C50, 53A30.

Key words and phrases. Lorentz conformal geometry, conformal Cartan connection, Einstein static universe, compact quotients of the Einstein universe, restricted conformal group of the Einstein universe, restricted conformal group of maximal dimension, Cartan domain of type IV.

Authors partially supported by PRIN 2015-2018 "Varietà reali e complesse: geometria, topologia e analisi armonica"; by the GNSAGA of INDAM; and by the FFABR Grant 2017 of MIUR. The present research was also partially supported by MIUR grant "Dipartimenti di Eccellenza" 20182022, CUP: E11G18000350001, DISMA, Politecnico di Torino. 
covering group $\widetilde{\mathrm{O}}_{+}^{\uparrow}(2, n+1)$ of $\mathrm{O}_{+}^{\uparrow}(2, n+1)$. In the following, $\widehat{\mathrm{O}}_{+}^{\uparrow}(2, n+1)$ will be referred to as the canonical covering of $\mathrm{O}_{+}^{\uparrow}(2, n+1)$. It is important to observe that: (1) both the Lie groups $\widehat{\mathrm{O}}_{+}^{\uparrow}(2, n+1)$ and $\widetilde{\mathrm{O}}_{+}^{\uparrow}(2, n+1)$ do not have faithful finite dimensional representations, so that there are no nice models for them as Lie groups of matrices 1 (2) the restricted conformal groups of $\mathcal{E}_{\mathrm{I}}^{1, n}, \mathcal{E}_{\mathrm{II}}^{1, n}$ ( $n$ odd), and $\mathcal{E}^{1, n}$ have the largest possible dimension for a Lorentz manifold of dimension $n+1$, namely $N=(n+3)(n+2) / 2$ (cf. Section 2 for more theoretical details on the restricted conformal group of a Lorentz manifold).

The purposes of this paper are twofold. The first is to provide explicit models for the Lie groups $\widehat{\mathrm{O}}_{+}^{\uparrow}(2, n+1)$ and $\widetilde{\mathrm{O}}_{+}^{\uparrow}(2, n+1)$, that is, to describe the underlying group manifolds and the respective group multiplications. The second purpose is to use the models for $\widehat{\mathrm{O}}_{+}^{\uparrow}(2, n+1)$ and $\widetilde{\mathrm{O}}_{+}^{\uparrow}(2, n+1)$ to address the question of the characterization of oriented, time-oriented, conformal Lorentz manifolds $\left(\mathbb{M}^{n+1},[g]\right)$ $(n \geq 2)$ whose restricted conformal group $\mathcal{C}_{+}^{\uparrow}(\mathbb{M})$ has maximal dimension.

The main results of the paper are presented in three theorems, which we now illustrate individually.

Theorem A provides explicit models for the canonical covering $\widehat{\mathrm{O}}_{+}^{\uparrow}(2, n+1)$ and for the universal covering $\widetilde{\mathrm{O}}_{+}^{\uparrow}(2, n+1)$. The underlying group manifolds are described explicitly as hypersurfaces in $\widehat{\mathrm{O}}_{+}^{\uparrow}(2, n+1) \times \mathbb{R}$ and in $\operatorname{Spin}(2, n+1) \times$ $\mathbb{R}$, and the respective group multiplications are given by single global formulas. Our approach was inspired by the basic construction of a manifold underlying the universal covering of a Lie group $G$ with $\pi_{1}(G)=\mathbb{Z}$ given in [29, and by the construction of nontrivial central extensions of the real symplectic group $\operatorname{Sp}(2 n, \mathbb{R})$, such as the circle extension $\operatorname{Mp}^{c}(2 n, \mathbb{R})$ and the universal covering group $\widetilde{\mathrm{Sp}}(2 n, \mathbb{R})$ (cf. 29, 30]). The idea of this method, in turn, has its origin in the classical work of Bargmann [4, 5] on the irreducible unitary representations of the Lorentz group.

Theorem B proves that the Lie group $\widehat{\mathrm{O}}_{+}^{\uparrow}(2, n+1)$ constructed in Theorem A is indeed isomorphic to the restricted conformal group of the Einstein static universe $\mathcal{E}^{1, n}$. It also describes the restricted conformal groups of two countably infinite series of compact quotients of $\mathcal{E}^{1, n}$, namely $\mathcal{E}_{\mathrm{I}, k}^{1, n}(k \geq 1)$ and $\mathcal{E}_{\mathrm{II}, k}^{1, n}(k \geq 0 ; n$ odd), called the integral compact forms of the first and second kind with index $k$, respectively. In particular, $\mathcal{E}_{\mathrm{I}, 1}^{1, n}=\mathcal{E}_{\mathrm{I}}^{1, n}$ and $\mathcal{E}_{\mathrm{II}, 0}^{1, n}=\mathcal{E}_{\mathrm{II}}^{1, n}$, which are referred to as the standard compact forms. Interestingly enough, the restricted conformal group of any integral compact form attains the maximum dimension $N$. It is important to observe that two integral compact forms with different indices in the same series, as well as two integral compact forms in different series, cannot be conformally equivalent. This is proved in Proposition 7.

Theorem $\mathrm{C}$ proves that if a connected, oriented, time-oriented, conformal Lorentz manifold of dimension $n+1(n \geq 2)$ has a restricted conformal group of maximal dimension $N$, then it is conformally equivalent to either $\mathcal{E}^{1, n}$ or to an integral compact form $\mathcal{E}_{\mathrm{I}, k}^{1, n}$ or $\mathcal{E}_{\mathrm{II}, k}^{1, n}$. The above characterization provides conformally nonequivalent geometric models for the Lorentz manifolds with an essential] restricted conformal

\footnotetext{
${ }^{1}$ In this respect, observe that $\widetilde{\mathrm{O}}_{+}^{\uparrow}(2,3) \cong \widetilde{\mathrm{Sp}}(4, \mathbb{R})$, the universal covering group of $\operatorname{Sp}(4, \mathbb{R})$.

${ }^{2}$ The conformal group of a Lorentz manifold $(\mathbb{M},[g])$ is said to be essential if it is strictly larger than the isometry group of any metric in the conformal class of $g$ (cf. 1, 2]).
} 
group of maximal dimension. Observe in particular that these models are all conformally flat. An interesting problem is to investigate the possibility of providing for the examples studied by Alekseevsky [3] suitable geometric models which are locally but not globally conformally equivalent to each other.

We conclude by recalling that in the Riemannian case, in contrast to the result of Theorem $\mathrm{C}$, a conformal Riemmannian manifold $(\mathbb{M},[g])$ of dimension $n \geq 3$ with a conformal group of maximal dimension must be conformally diffeomorphic to $\mathbb{S}^{n}$ with its natural conformal structure (cf. [21, 23, 26]).

The paper is organized as follows. Section 2 collects and reformulates some known facts about conformal Lorentz geometry for the purpose of the discussion. More specifically, it describes the Einstein static universe and its compact quotients, and among the compact quotients distinguishes two countably infinite families, the integral compact forms of the first and second kind. The construction of the Cartan conformal bundle and of the normal conformal Cartan connection for an oriented, time-oriented, conformal Lorentz manifold $\mathbb{M}$ of dimension $n+1 \geq 3$ is then briefly recalled. A classical result of Cartan and Kobayashi on the conformal group of a Riemannian manifold (cf. [11, 21]) is then extended to the case of the restricted conformal group of a Lorentz manifold. This technical result, which was indeed largely predictable, will play an important role in the proofs of Theorems A and B. Finally, the special examples $\mathcal{E}_{\mathrm{I}}^{1, n}$ and $\mathcal{E}_{\mathrm{II}}^{1, n}$ are discussed.

Section 3 constructs the canonical covering $\widehat{\mathrm{O}}_{+}^{\uparrow}(2, n+1)$ of $\mathrm{O}_{+}^{\uparrow}(2, n+1)$ using the transitive action of $\mathrm{O}_{+}^{\uparrow}(2, n+1)$ on the irreducible bounded symmetric domain of type IV, regarded as a homogeneous space of $(n+1) \times 2$ real matrices (cf. [7, 10, 17, 18, 19, 31). The group manifold of the canonical covering is realized explicitly as an embedded submanifold of the product $\mathrm{O}_{+}^{\uparrow}(2, n+1) \times \mathbb{R}$ and the group multiplication defining the Lie group structure is given by a single global formula. The center of $\widehat{\mathrm{O}}_{+}^{\uparrow}(2, n+1)$ is also computed. This is the content of Theorem $\mathrm{A}$ By a similar construction, an explicit realization of the universal covering group of $\mathrm{O}_{+}^{\uparrow}(2, n+1)$ is obtained. We then prove that $\widehat{\mathrm{O}}_{+}^{\uparrow}(2, n+1)$ is isomorphic to the restricted conformal group of the Einstein static universe and describe the restricted conformal groups of the integral compact forms. This is the content of Theorem B

Section 4 proves that if the restricted conformal group of an oriented, timeoriented Lorentz manifold $\mathbb{M}$ has maximal dimension, then $\mathbb{M}$ is conformally equivalent to either the simply connected Einstein static universe, or to one of its integral compact forms. This is the content of Theorem $\mathrm{C}$

\section{The Einstein Static Universe And the integral COMPaCt FORMS}

In this section we introduce two infinite series of compact quotients of $\mathcal{E}^{1, n}$, the so-called integral compact forms of the first and second kind. These include as special cases the compact Einstein universes $\mathcal{E}_{\mathrm{I}}^{1, n}$ and $\mathcal{E}_{\mathrm{II}}^{1, n}$ ( $n$ odd), also called standard compact forms of the first and second kind. We then present a direct construction of the Cartan conformal bundle and of the normal conformal Cartan connection for a conformal Lorentz manifold without resorting to the abstract theory of prolongation of $G$-structures. We reformulate, for conformal Lorentz manifolds, a classical result on the conformal group of a Riemannian manifold. As an example, we describe the restricted conformal groups of the standard forms $\mathcal{E}_{\mathrm{I}}^{1, n}$ and $\mathcal{E}_{\mathrm{II}}^{1, n}$ ( $n$ odd). 
2.1. Preliminaries. For given integers $p, q, 1 \leq p \leq 2, p<q$, let $\mathbb{R}^{p, q}$ denote $\mathbb{R}^{m}$, $m=p+q$, with the nondegenerate scalar product

$$
\langle x, y\rangle=-x^{0} y^{0}+(-1)^{p-1} x^{1} y^{1}+\sum_{j=2}^{m-1} x^{j} y^{j}={ }^{t} x G y
$$

of signature $(p, q)$, where $x^{0}, \ldots, x^{m-1}$ denote the coordinates with respect to the standard basis $e=\left(e_{0}, \ldots, e_{m-1}\right)$ of $\mathbb{R}^{m}$. Let $\mathrm{O}_{+}^{\uparrow}(p, q)$ denote the identity component of the pseudo-orthogonal group of (2.1). For the purpose of conformal geometry, instead of the canonical coordinates, it is convenient to use the coordinates $u={ }^{t}\left(u^{0}, \ldots, u^{m-1}\right)$ defined by $u=\mathcal{D}_{p} x$, where $\mathcal{D}_{p} \in \mathrm{GL}(m, \mathbb{R})$ is given by

$$
\sqrt{2} \mathcal{D}_{p}:=E_{0}^{0}+(-1)^{p-1} E_{m-1}^{0}+E_{0}^{m-1}-(-1)^{p-1} E_{m-1}^{m-1}+\sqrt{2} \sum_{j=1}^{m-2} E_{j}^{j} .
$$

Here $E_{k}^{h}, 0 \leq h, k \leq m-1$, denotes the elementary $m \times m$ matrix with 1 in the $(h, k)$ place and zero elsewhere. In the coordinates $u={ }^{t}\left(u^{0}, \ldots, u^{m-1}\right)$ the scalar product (2.1) takes the form

$$
-u^{0} v^{m-1}-u^{m-1} v^{0}+(-1)^{p-1} u^{1} v^{1}+\sum_{j=2}^{m-2} u^{j} v^{j} .
$$

The pseudo-orthogonal group $\mathrm{M}_{+}^{\uparrow}(p, q)$ of (2.2) is the image of the faithful representation of $\mathrm{O}_{+}^{\uparrow}(p, q)$ given by

$$
\chi: \mathrm{O}_{+}^{\uparrow}(p, q) \ni \mathbf{X} \longmapsto \mathcal{D}_{p} \mathbf{X} \mathcal{D}_{p}^{-1} \in \mathrm{GL}(m, \mathbb{R}),
$$

that is, $\mathrm{M}_{+}^{\uparrow}(p, q)=\mathcal{D}_{p} \mathrm{O}_{+}^{\uparrow}(p, q) \mathcal{D}_{p}^{-1}$. The Lie algebra of $\mathrm{M}_{+}^{\uparrow}(p, q)$ will be denoted by $\mathfrak{m}(p, q)$.

Let $\mathrm{H}_{+}^{\uparrow}(p, q) \subset \mathrm{M}_{+}^{\uparrow}(p, q)$ be the parabolic subgroup

$$
\mathrm{H}_{+}^{\uparrow}(p, q):=\left\{\mathbf{X} \in \mathrm{M}_{+}^{\uparrow}(p, q) \mid \mathbf{X} e_{0}=r e_{0}, r \in \mathbb{R}, r>0\right\} .
$$

The elements of $\mathrm{H}_{+}^{\uparrow}(p, q)$ can be written as

$$
\mathbf{X}(r, B, y)=\left(\begin{array}{ccc}
r & { }^{*} y \mathrm{~B} & { }^{*} y y / 2 r \\
0 & \mathrm{~B} & y / r \\
0 & 0 & r^{-1}
\end{array}\right)
$$

where $r>0, y={ }^{t}\left(y^{1}, \ldots, y^{m-2}\right) \in \mathbb{R}^{m-2}, \mathrm{~B} \in \mathrm{O}_{+}^{\uparrow}(p-1, q-1)$ and ${ }^{*} y=$ $\left((-1)^{p-1} y^{1}, y^{2}, \ldots, y^{m-2}\right) \in\left(\mathbb{R}^{m-2}\right)^{*} \sqrt{3}$ Let $\mathfrak{h}(p, q)$ be the Lie algebra of $\mathrm{H}_{+}^{\uparrow}(p, q)$.

2.2. The Einstein static universe. The $(1+n)$-dimensional Einstein static universe $\mathcal{E}^{1, n}$ is the hypersurface of $\mathbb{R}^{1, n+1} \cong \mathbb{R} \times \mathbb{R}^{n+1}$ defined by

$$
\mathcal{E}^{1, n}:=\left\{(\tau, x) \in \mathbb{R}^{1, n+1} \mid{ }^{t} x x=1\right\} \cong \mathbb{R} \times \mathbb{S}^{n},
$$

equipped with the Lorentz metric

$$
\widehat{\ell}_{\mathcal{E}}=-d \tau^{2}+\imath^{*}\left(\sum_{j=1}^{n+1}\left(d x^{j}\right)^{2}\right)
$$

\footnotetext{
${ }^{3}$ If $p=1$, then $\mathrm{O}_{+}^{\uparrow}(p-1, q-1)$ is the special orthogonal group $\mathrm{SO}(q-1)$.
} 
where $\imath: \mathbb{S}^{n} \hookrightarrow \mathbb{R}^{n+1}$ denotes the inclusion map. On $\mathcal{E}^{1, n}$ we consider the time-orientation given by requiring that the unit timelike vector field $\partial_{\tau}$ is futuredirected, and the orientation induced by the volume form

$$
\left.\Omega_{\widehat{\mathcal{E}}}\right|_{(\tau, x)}=\left.\left(d \tau \wedge \imath_{x}^{*}\left(d x^{1} \wedge \cdots \wedge d x^{n+1}\right)\right)\right|_{T_{(\tau, x)}\left(\mathcal{E}^{1, n}\right)} .
$$

The motivation for this terminology is that $\mathcal{E}^{1, n}$ is a static solution of Einstein's equation with a positive cosmological constant [13, 16. This solution was proposed by Einstein himself as a model of a closed universe filled with a perfect fluid of constant pressure and energy density [15. The physical relevance of the Einstein universe is due to the fact that every Friedmann-Robertson-Walker spacetime can be conformally embedded in $\mathcal{E}^{1, n}$ [16].

2.3. Compact quotients: The integral compact forms. Let $\tau_{*}>0$ be a positive real number. The subgroup $\mathcal{T}_{\tau^{*}}$ of the isometry group $\mathbb{R} \times \mathrm{SO}(n+1)$ generated by the translation $\mathrm{T}_{\tau_{*}}:(\tau, \mathrm{y}) \mapsto\left(\tau+\tau_{*}, \mathrm{y}\right)$ acts properly discontinuously on $\mathcal{E}^{1, n}$. This action preserves the Lorentz metric $\widehat{\ell}_{\mathcal{E}}$, the volume element $\Omega_{\widehat{\mathcal{E}}}$, and the time-orientation. The quotient manifold $\mathcal{E}^{1, n} / \mathcal{T}_{\tau^{*}}$ possesses a unique Lorentz metric $\ell_{\mathcal{E}, \tau_{*}}$, and a unique orientation and time-orientation, such that the covering map $\pi_{\tau_{*}}: \mathcal{E}^{1, n} \rightarrow \mathcal{E}^{1, n} / \mathcal{T}_{\tau^{*}}$ is a local isometry preserving orientation and timeorientation.

Definition 1. If $\tau_{*}=2 k \pi, k$ a positive integer, the quotient manifold $\mathcal{E}^{1, n} / \mathcal{T}_{\tau^{*}}$, denoted by $\mathcal{E}_{\mathrm{I}, k}^{1, n}$, is referred to as the integral compact form of the first kind with index $k$. In this case, the covering $\pi_{\tau_{*}}$ will be denoted by $\pi_{\mathrm{I}, k}$. When $k=1$, the integral compact form $\mathcal{E}_{\mathrm{I}, 1}^{1, n}$ coincides with $\mathcal{E}_{\mathrm{I}}^{1, n}$, and is referred to as the standard compact form of the first kind. The integral forms $\mathcal{E}_{\mathrm{I}, k}^{1, n}$ are all diffeomorphic to $\mathbb{S}^{1} \times \mathbb{S}^{n} \subset \mathbb{C} \times \mathbb{R}^{n+1}$.

Remark 1 . The standard compact form $\mathcal{E}_{\mathrm{I}}^{1, n}=(\mathbb{R} / 2 \pi \mathbb{Z}) \times \mathbb{S}^{n} \cong \mathbb{S}^{1} \times \mathbb{S}^{n}$. Here $\mathbb{S}^{1}$ is the unit circle viewed as a multiplicative subgroup of $\mathbb{C}$, and the isomorphism $\mathbb{R} / 2 \pi \mathbb{Z} \cong \mathbb{S}^{1}$ is induced by $t \mapsto e^{i t}$. The conformal structure on $\mathcal{E}_{\mathrm{I}}^{1, n}$ is given by the Lorentz metric $-d \theta^{2}+g_{\mathbb{S}^{n}}$, where $\theta: \mathbb{R} / 2 \pi \mathbb{Z} \rightarrow \mathbb{S}^{1}$ is the argument function. For a positive integer $k$, the map $p_{k}: \mathbb{S}^{1} \times \mathbb{S}^{n} \ni(\mathrm{x}, \mathrm{y}) \longmapsto\left(\mathrm{x}^{k}, \mathrm{y}\right) \in \mathbb{S}^{1} \times \mathbb{S}^{n}$ defines a $k: 1$ covering. Therefore, the integral form $\mathcal{E}_{\mathrm{I}, k}^{1, n}$ can be thought of as $\mathbb{S}^{1} \times \mathbb{S}^{n}$ with the conformal structure induced by the Lorentz metric $\ell_{\mathcal{E}, k}=p_{k}{ }^{*}\left(-d \theta^{2}+g_{\mathbb{S}^{n}}\right)=$ $-k^{2} d \theta^{2}+g_{\mathbb{S}^{n}}$. Notice that $p_{k}: \mathcal{E}_{\mathrm{I}, k}^{1, n} \rightarrow \mathcal{E}_{\mathrm{I}}^{1, n}$ is a $k: 1$ Lorentzian covering map.

If $n$ is odd, the map $\mathrm{T}_{\tau_{*}}^{\prime}:(\tau, \mathrm{y}) \mapsto\left(\tau+\tau_{*},-\mathrm{y}\right)$ is an isometry that preserves the orientation and the time-orientation. The subgroup $\mathcal{T}_{\tau^{*}}^{\prime}$ generated by $\mathrm{T}_{\tau_{*}}^{\prime}$ acts properly discontinuously on $\mathcal{E}^{1, n}$. Consequently, the quotient manifold $\mathcal{E}^{1, n} / \mathcal{T}_{\tau^{*}}^{\prime}$ inherits a unique Lorentz metric $\ell_{\mathcal{E}, \tau_{*}}^{\prime}$ and a unique orientation and time-orientation such that the covering map $\pi_{\tau_{*}}^{\prime}: \mathcal{E}^{1, n} \rightarrow \mathcal{E}^{1, n} / \mathcal{T}_{\tau^{*}}^{\prime}$ is a local isometry preserving orientation and time-orientation.

Definition 2. If $n$ is odd and $\tau_{*}=(2 k+1) \pi, k$ a non-negative integer, the quotient manifold $\mathcal{E}^{1, n} / \mathcal{T}_{\tau^{*}}^{\prime}$, denoted by $\mathcal{E}_{\mathrm{II}, k}^{1, n}$, is called the integral compact form of the second kind with index $k$. In this case, the covering $\pi_{\tau_{*}}^{\prime}$ will be denoted by $\pi_{\mathrm{II}, k}$. When $k=0$, the integral compact form $\mathcal{E}_{\mathrm{II}, 0}^{1, n}$ coincides with $\mathcal{E}_{\mathrm{II}}^{1, n}$, and is referred to as the standard compact form of the second kind. Notice that also the integral forms $\mathcal{E}_{\mathrm{II}, k}^{1, n}$ are diffeomorphic to $\mathbb{S}^{1} \times \mathbb{S}^{n}$. If $n$ is even, this is not true anymore. 
2.4. The Cartan conformal bundle. Let us start by recalling some definitions and fixing some notation.

Definition 3. Two Lorentz metrics $g$ and $g^{\prime}$ on a manifold $\mathbb{M}$ are said to be conformal to each other if $g^{\prime}=r^{2} g$, for some smooth function $r: \mathbb{M} \rightarrow \mathbb{R}$. The conformal class of $g$ is $[g]=\left\{g^{\prime} \mid g^{\prime}\right.$ is conformal to $\left.g\right\}$. A conformal Lorentz structure on $\mathbb{M}$ amounts to the assignment of a conformal class $[g]$ of Lorentz metrics on $\mathbb{M}$.

A conformal Lorentz manifold $(\mathbb{M},[g])$ is an oriented, connected smooth manifold $\mathbb{M}$ of dimension $n+1 \geq 3$ with a conformal Lorentz structure $[g]$. We assume that the conformal structure is time-orientable, i.e., that the bundle $\dot{N}(\mathbb{M})$ of nonzero timelike tangent vectors is disconnected. A time-orientation is defined by the choice of a connected component $\dot{\mathrm{N}}^{\uparrow}(\mathbb{M})$ of $\dot{\mathrm{N}}(\mathbb{M})$.

A conformal transformation of $(\mathbb{M},[g])$ is a diffeomorphism $F: \mathbb{M} \rightarrow \mathbb{M}$ that preserves the conformal class $[g]$, that is, $F^{*}([g])=[g]$. A restricted conformal transformation is a conformal transformation which, in addition, preserves the orientation and the time-orientation. Let $\mathcal{C}_{+}^{\uparrow}(\mathbb{M})$ denote the group of all restricted conformal transformations of $(\mathbb{M},[g])$. We call $\mathcal{C}_{+}^{\uparrow}(\mathbb{M})$ the restricted conformal group of $\mathbb{M}$. It is a classical result that $\mathcal{C}_{+}^{\uparrow}(\mathbb{M})$ is a Lie transformation group.

Let $\mathcal{A}=\left(A_{1}, \ldots, A_{n+1}\right)$ be a positive basis of the tangent space $T_{p}(\mathbb{M})$ at a point $p \in \mathbb{M}$ and let $\left(A^{1}, \ldots, A^{n+1}\right)$ be its dual basis. We say that $\mathcal{A}$ is a positive linear conformal frame at $p \in \mathbb{M}$ if

$$
\left.-A^{1} \odot A^{1}+\sum_{j=2}^{n+1} A^{j} \odot A^{j} \in[g]_{\left.\right|_{p}}, \quad A_{1} \in \dot{\mathrm{N}}^{\uparrow}(\mathbb{M})\right)_{\left.\right|_{p}} .
$$

The set $\mathrm{CO}_{+}^{\uparrow}(\mathbb{M})$ of all positive linear conformal frames at all points of $\mathbb{M}$ defines a principal fiber bundle over $\mathbb{M}, \pi_{\mathrm{C}}: \mathrm{CO}_{+}^{\uparrow}(\mathbb{M}) \rightarrow \mathbb{M}$, with structure group

$$
\mathrm{CO}_{+}^{\uparrow}(1, n)=\left\{r \mathrm{~B} \mid r>0, \mathrm{~B} \in \mathrm{O}_{+}^{\uparrow}(1, n)\right\} .
$$

As a subbundle of the bundle $\mathrm{L}(\mathbb{M})$ of linear frames over $\mathbb{M}, \mathrm{CO}_{+}^{\uparrow}(\mathbb{M})$ defines a $\mathrm{CO}_{+}^{\uparrow}(1, n)$-structure on $\mathbb{M}$. By a positive linear conformal frame field is meant a local section of $\pi_{\mathrm{C}}$. Let $\mathcal{C} \mathcal{O}_{+}^{\uparrow}=\left\{\mathcal{A}^{\alpha}\right\}_{\alpha \in \mathcal{C}}$ denote the sheaf of positive linear conformal frame fields. For each $\alpha \in \mathcal{C}$, let $U_{\alpha} \subset \mathbb{M}$ be the domain of definition of $\mathcal{A}^{\alpha}$. Let $\mathcal{C}_{2}=\left\{(\alpha, \beta) \in \mathcal{C} \times \mathcal{C} \mid U_{\alpha} \cap U_{\beta} \neq \emptyset\right\}$, and let $\Gamma=\left\{\mathrm{A}_{\alpha}^{\beta}\right\}_{(\alpha, \beta) \in \mathcal{C}_{2}}$ denote the Cech 1-cocycle defined by the transition functions of $\mathrm{CO}_{+}^{\uparrow}(\mathbb{M})$. For each $\mathcal{A}^{\alpha} \in \mathcal{C} \mathcal{O}_{+}^{\uparrow}$, let $\omega_{\alpha}={ }^{t}\left(\omega_{\alpha}^{1}, \ldots, \omega_{\alpha}^{n+1}\right)$ be the corresponding dual coframe. Then, if $(\alpha, \beta) \in \mathcal{C}_{2}$,

$$
\mathcal{A}^{\beta}=\frac{1}{r_{\alpha}^{\beta}} \mathcal{A}^{\alpha} \mathrm{B}_{\alpha}^{\beta}
$$

where $r_{\alpha}^{\beta}: U_{\alpha} \cap U_{\beta} \rightarrow \mathbb{R}^{+}$and $\mathrm{B}_{\alpha}^{\beta}: U_{\alpha} \cap U_{\beta} \rightarrow \mathrm{O}_{+}^{\uparrow}(1, n)$ A Define

$$
y_{\alpha}^{\beta}={ }^{t}\left(y_{\alpha}^{\beta, 1}, y_{\alpha}^{\beta, 2}, \ldots, y_{\alpha}^{\beta, n+1}\right): U_{\alpha} \cap U_{\beta} \rightarrow \mathbb{R}^{1, n}
$$

by requiring that

and put

$$
d \log r_{\alpha}^{\beta}=\left(\omega_{\alpha}^{1}, \ldots, \omega_{\alpha}^{n+1}\right) y_{\alpha}^{\beta}
$$

$$
{ }^{*} y_{\alpha}^{\beta}=\left(-y_{\alpha}^{\beta, 1}, y_{\alpha}^{\beta, 2}, \ldots, y_{\alpha}^{\beta, n+1}\right) .
$$

\footnotetext{
${ }^{4}$ Here $\mathbb{R}^{+}=\{r \in \mathbb{R} \mid r>0\}$.
} 
Let

$$
\dot{\mathrm{A}}_{\alpha}^{\beta}: U_{\alpha} \cap U_{\beta} \rightarrow \mathrm{H}_{+}^{\uparrow}(2, n+1)
$$

be the smooth $\mathrm{H}_{+}^{\uparrow}(2, n+1)$-valued map defined by

$$
\dot{\mathrm{A}}_{\alpha}^{\beta}=\left(\begin{array}{ccc}
r_{\alpha}^{\beta} & { }^{*} y_{\alpha}^{\beta} \mathrm{B}_{\alpha}^{\beta} & { }^{*} y_{\alpha}^{\beta} y_{\alpha}^{\beta} / 2 r_{\alpha}^{\beta} \\
0 & \mathrm{~B}_{\alpha}^{\beta} & y_{\alpha}^{\beta} / r_{\alpha}^{\beta} \\
0 & 0 & 1 / r_{\alpha}^{\beta}
\end{array}\right)
$$

It is now a computational matter to check that $\dot{\Gamma}=\left\{\dot{\mathrm{A}}_{\alpha}^{\beta}\right\}_{(\alpha, \beta) \in \mathcal{C}_{2}}$ defines a C̆ech 1cocycle with values in $\mathrm{H}_{+}^{\uparrow}(2, n+1)$ on the given covering of $\mathbb{M}$. Consequently, see for instance [20, Proposition 5.2, page 52], there exists a unique principal fiber bundle $\pi_{\mathrm{Q}}: \mathrm{Q}(\mathbb{M}) \rightarrow \mathbb{M}$, with structure group $\mathrm{H}_{+}^{\uparrow}(2, n+1)$, admitting $\dot{\Gamma}$ as a 1-cocycle of transition functions and with an atlas $\dot{\mathcal{O}}_{+}^{\uparrow}=\left\{\dot{\mathcal{A}}^{\alpha}\right\}_{\alpha \in \mathcal{C}}$ of local sections.

Definition 4. We call $\mathrm{Q}(\mathbb{M})$ the (restricted) Cartan conformal bundle of $\mathbb{M}$.

Remark 2. The construction of $\mathrm{Q}(\mathbb{M})$ from $\mathrm{CO}_{+}^{\uparrow}(\mathbb{M})$ is a particular instance of the procedure known as prolongation of a $G$-structure (see [21]). Starting from a $G$-structure $P \subset \mathrm{L}(\mathbb{M})$, there is a canonical way to construct a $G^{1}$-structure $P^{1} \subset \mathrm{L}(P)$. This construction can be repeated on $P^{1}$ to obtain a $G^{2}$-structure $P^{2}$, and so on. If $G \subset \operatorname{GL}(M, \mathbb{R})$ is a Lie subgroup of finite order, say $k$, then $G^{k}=\{e\}$, and the $k$ th prolongation $P^{k}$ is an $\{e\}$-structure. The bundle $P_{k-1}$ encodes all relevant pieces of information about the local geometry of the $G$-structure. Usually, geometries of infinite order (e.g., complex, contact or symplectic geometries) do not have local invariants. In the case at hand, $\mathrm{Q}(\mathbb{M})=\mathrm{CO}_{+}^{\uparrow}(\mathbb{M})^{1}$ and $\mathrm{Q}(\mathbb{M})^{1}$ is an $\{e\}$ structure on $\mathrm{Q}(\mathbb{M})$, since the group $\mathrm{CO}_{+}^{\uparrow}(1, n)$ has order 2 .

2.5. The normal conformal connection. Let $\mathcal{A}^{\alpha}$ be a positive linear conformal frame field of $\mathbb{M}$ defined on $U_{\alpha}$ and $\omega_{\alpha}={ }^{t}\left(\omega_{\alpha}^{1}, \ldots, \omega_{\alpha}^{n+1}\right)$ the corresponding dual coframe field. Then the quadratic form

$$
\ell_{\alpha}=-\omega_{\alpha}^{1} \odot \omega_{\alpha}^{1}+\sum_{j=2}^{n+1} \omega_{\alpha}^{j} \odot \omega_{\alpha}^{j}=\sum_{i, j=1}^{n+1} \widetilde{\delta}_{i j} \omega_{\alpha}^{i} \odot \omega_{\alpha}^{j}, \quad \widetilde{\delta}_{i j}=\widetilde{\delta}_{j i}, 1 \leq i, j \leq n+1
$$

belongs to the conformal class $[g]$ on $U_{\alpha}$. Consequently, there exists a unique $\mathfrak{o}(1, n)$ valued exterior differential 1-form $\theta_{\alpha}=\left(\theta_{\alpha j}^{i}\right) \in \Omega^{1}\left(U_{\alpha}\right) \otimes \mathfrak{o}(1, n)$, the Levi-Civita connection form of $\ell_{\alpha}$ with respect to the pseudo-orthogonal frame field $\mathcal{A}^{\alpha}$, such that

$$
d \omega_{\alpha}=-\theta_{\alpha} \wedge \omega_{\alpha} .
$$

Consider the curvature form $\Theta_{\alpha} \in \Omega^{2}\left(U_{\alpha}\right) \otimes \mathfrak{o}(1, n)$ defined by

$$
\Theta_{\alpha}=d \theta_{\alpha}+\theta_{\alpha} \wedge \theta_{\alpha}
$$

We write

$$
\Theta_{\alpha j}^{i}=\frac{1}{2} \sum_{h, k=1}^{n+1}\left(\mathrm{R}_{\alpha}\right)_{j h k}^{i} \omega_{\alpha}^{h} \wedge \omega_{\alpha}^{k}
$$

where the functions $\left(\mathrm{R}_{\alpha}\right)_{j h k}^{i}$ are the local components of the Riemann curvature tensor of $\ell_{\alpha}$ with respect to $\mathcal{A}^{\alpha}$. The Ricci tensor components $\left(\mathrm{R}_{\alpha}\right)_{j h}$ and the 
scalar curvature $\mathrm{R}_{\alpha}$ are given by

$$
\left(\mathrm{R}_{\alpha}\right)_{j h}=\sum_{k=1}^{n+1}\left(\mathrm{R}_{\alpha}\right)_{j h k}^{k}, \quad \mathrm{R}_{\alpha}=\sum_{h=1}^{n+1}\left(\mathrm{R}_{\alpha}\right)_{h h} .
$$

Let $\eta_{\alpha}=\left(\eta_{\alpha, 1}, \ldots, \eta_{\alpha, n+1}\right) \in \Omega^{1}\left(U_{\alpha}\right) \otimes\left(\mathbb{R}^{1, n}\right)^{*}$ be the vector-valued 1-form defined by

$$
\eta_{\alpha, j}=\frac{\mathrm{R}_{\alpha}}{2 n(n-1)} \sum_{h=1}^{n+1} \widetilde{\delta}_{j h} \omega_{\alpha}^{h}-\frac{1}{n-1} \sum_{h=1}^{n+1}\left(\mathrm{R}_{\alpha}\right)_{j h} \omega_{\alpha}^{h}, \quad j=1, \ldots, n+1 .
$$

Next, let

$$
{ }^{*} \omega_{\alpha}=\left(-\omega_{\alpha}^{1}, \omega_{\alpha}^{2}, \ldots, \omega_{\alpha}^{n+1}\right), \quad{ }^{*} \eta_{\alpha}={ }^{t}\left(-\eta_{\alpha, 1}, \ldots, \eta_{\alpha, n+1}\right)
$$

and consider the $\mathfrak{m}(2, n+1)$-valued 1 -form $\phi_{\alpha}$ given by

$$
\phi_{\alpha}=\left(\begin{array}{ccc}
0 & \eta_{\alpha} & 0 \\
\omega_{\alpha} & \theta_{\alpha} & { }^{*} \eta_{\alpha} \\
0 & { }^{*} \omega_{\alpha} & 0
\end{array}\right)
$$

By similar calculations as those performed in the Riemannian case in 24, Chapter 1 , Section 4] for determining the transformation rules, under a conformal metric change, of the components of the Levi-Civita connection and of the modified Ricci tensor in the expression of $\eta_{\alpha, j}$, it can be verified that if $\mathcal{A}^{\alpha}=\mathcal{A}^{\beta} \mathrm{A}_{\beta}^{\alpha}$, then

$$
\phi_{\alpha}=\left(\dot{\mathrm{A}}_{\beta}^{\alpha}\right)^{-1} \phi_{\beta} \dot{\mathrm{A}}_{\beta}^{\alpha}+\left(\dot{\mathrm{A}}_{\beta}^{\alpha}\right)^{-1} d \dot{\mathrm{A}}_{\beta}^{\alpha} .
$$

According to [20, Proposition 1.4, page 66], we can thus state the following.

Proposition 1. There exists a unique exterior differential 1-form $\phi \in \Omega^{1}[\mathrm{Q}(\mathbb{M})]$ $\otimes \mathfrak{m}(2, n+1)$ such that:

- if $\xi \in \mathfrak{h}(2, n+1)$, then $\phi\left(\xi^{*}\right)=\xi$ 占

- $R_{\mathrm{X}}^{*}(\phi)=\mathrm{X}^{-1} \phi \mathrm{X}$, for every $\mathrm{X} \in \mathrm{H}_{+}^{\uparrow}(2, n+1)$;

- $\dot{\mathcal{A}}_{\alpha}^{*}(\phi)=\phi_{\alpha}, \forall \dot{\mathcal{A}}_{\alpha} \in \dot{\mathcal{C O}} \dot{O}_{+}^{\uparrow}$.

Definition 5. The 1-form $\phi$ is the normal conformal Cartan connection of the conformal Lorentz manifold $\mathbb{M}$.

Remark 3. Using block matrix notation, the normal connection takes the form

$$
\phi=\left(\begin{array}{ccc}
\phi_{0}^{0} & \phi^{0} & 0 \\
\phi_{0} & \widehat{\phi} & { }^{*} \phi^{0} \\
0 & { }^{t} \phi_{0} & -\phi_{0}^{0}
\end{array}\right),
$$

where $\phi_{0}^{0}$ is a scalar 1-form, $\phi_{0}={ }^{t}\left(\phi_{0}^{1}, \ldots, \phi_{0}^{n+1}\right)$ and $\phi^{0}=\left(\phi_{1}^{0}, \ldots, \phi_{n+1}^{0}\right)$ are vectorvalued 1 -forms, and $\widehat{\phi}=\left(\phi_{j}^{i}\right), i, j=1, \ldots, n+1$, is a 1 -form taking values in the Lie algebra $\mathfrak{o}(1, n)$. The connection form $\phi$ satisfies the following basic properties.

(1) The 1 -forms $\phi_{0}^{0}, \phi_{0}^{1}, \ldots, \phi_{0}^{n+1}, \phi_{1}^{0}, \ldots, \phi_{n+1}^{0}, \phi_{j}^{i}, 1 \leq i<j \leq n+1$, are linearly independent and define an absolute parallelism on $\mathrm{Q}(\mathbb{M})$, the normal parallelism of the Cartan conformal bundle.

(2) The 1 -forms $\phi_{0}^{1}, \ldots, \phi_{0}^{n+1}$ are semibasic 6

\footnotetext{
${ }^{5}$ Here $\xi^{*}$ stands for the fundamental vector field on $\mathrm{Q}(\mathbb{M})$ generated by $\xi$.

${ }^{6} \mathrm{~A}$ form is semibasic of it annihilates the vertical vector fields.
} 
(3) For every restricted conformal transformation $F: \mathbb{M} \rightarrow \mathbb{M}$, there exists a unique lifted diffeomorphism $\dot{F}: \mathrm{Q}(\mathbb{M}) \rightarrow \mathrm{Q}(\mathbb{M})$, called the conformal prolongation of $F$, that preserves the normal conformal connection $\phi$, i.e., $(\dot{F})^{*}(\phi)=\phi$. Conversely, any diffeomorphism $\mathcal{F}$ of $\mathrm{Q}(\mathbb{M})$ that preserves $\phi$ arises in this way, that is, there exists a unique restricted conformal transformation $F$ of $\mathbb{M}$ such that $\mathcal{F}=\dot{F}$. In particular, if $\operatorname{Aut}(\phi)$ denotes the group of diffeomorphisms of $\mathrm{Q}(\mathbb{M})$ preserving $\phi$, then the mapping $F \in \mathcal{C}_{+}^{\uparrow}(\mathbb{M}) \mapsto \dot{F} \in \operatorname{Aut}(\phi)$ is an isomorphism.

2.6. The restricted conformal group of a Lorentzian manifold. We are now in a position to apply to $\mathrm{Q}(\mathbb{M})$, with the normal parallelism induced by the normal conformal connection, a classical result of S. Kobayashi on the transformation group of a manifold with an absolute parallelism [21, Chapter I, Theorem 3.2].

Theorem 2 (S. Kobayashi). Let $N$ be an $n$-dimensional differentiable manifold with an absolute parallelism, i.e., a coframe $\left\{\eta^{1}, \ldots, \eta^{n}\right\}$ of globally defined 1-forms which are linearly independent at each point of $N$. Let $G=\operatorname{Aut}\left(\left\{\eta^{i}\right\}\right)$ be the group of automorphisms of the absolute parallelism, i.e., the group of diffeomorphisms $F: N \rightarrow N$, such that $F^{*}\left(\eta^{i}\right)=\eta^{i}, i=1, \ldots, n$. Then, $G$ is a Lie transformation group such that $\operatorname{dim} \mathrm{G} \leq n$. More precisely, for any $p \in N$, the mapping $F \in G \mapsto$ $F(p) \in N$ is injective and its image $\{F(p) \mid F \in G\}$ is a closed submanifold of $N$. The submanifold structure on this image makes $G$ into a Lie transformation group.

Accordingly, taking into account the above discussion, we can state the following.

Theorem 3. Let $\mathbb{M}$ be an oriented, time-oriented conformal Lorentz manifold of dimension $n+1 \geq 3$. Let $L: G \times \mathbb{M} \rightarrow \mathbb{M}$ be an effective left action of a connected Lie group $\mathrm{G}$ on $\mathbb{M}$ by restricted conformal transformations. For any $\dot{\mathcal{A}}_{*} \in \mathrm{Q}(\mathbb{M})$, the mapping

$$
\mathfrak{j}: \mathrm{G} \ni g \longmapsto \dot{L}_{g}\left(\dot{\mathcal{A}}_{*}\right) \in \mathrm{Q}(\mathbb{M})
$$

is a 1-1 immersion of $\mathrm{G}$ into $\mathrm{Q}(\mathrm{M})$. Here, for each $g \in \mathrm{G}, \dot{L}_{g}$ denotes the conformal prolongation of $L_{g}$ (cf. Remark 3$)$. In particular, $\operatorname{dim} \mathrm{G} \leq \operatorname{dim} \mathrm{Q}(\mathbb{M})=\frac{1}{2}(n+3)(n+$ 2). If $\operatorname{dim} \mathrm{G}=\frac{1}{2}(n+3)(n+2)$, then:

(1) The mapping $\mathfrak{j}$ is a diffeomorphism and $\mathrm{Q}(\mathbb{M})$ inherits from $\mathrm{G}$ the structure of a Lie group with neutral element $\dot{\mathcal{A}}_{*}$. Moreover, $\mathrm{G}$ and $\mathrm{Q}(\mathbb{M})$ are isomorphic to $\mathcal{C}_{+}^{\uparrow}(\mathbb{M})$, the restricted conformal group of $\mathbb{M}$.

(2) $\mathrm{Q}(\mathbb{M})$ is locally isomorphic to $\mathrm{M}_{+}^{\uparrow}(2, n+1)$.

(3) If $p_{*}=\pi_{\mathrm{Q}}\left(\dot{\mathcal{A}}_{*}\right)$, then the fiber $\pi_{\mathrm{Q}}^{-1}\left(p_{*}\right)$ is a connected Lie subgroup isomorphic to $\mathrm{H}_{+}^{\uparrow}(2, n+1)$.

(4) $\pi_{\mathrm{Q}}^{-1}\left(p_{*}\right)$ is the stabilizer of the point $p_{*}$ for the action of $\mathrm{Q}(\mathbb{M})$ on $\mathbb{M}$.

(5) $\pi_{\mathrm{Q}}^{-1}\left(p_{*}\right)$ is the maximal integral submanifold through $\dot{\mathcal{A}}_{*}$ of the left-invariant, completely integrable Pfaffian differential system generated by the 1-forms $\phi_{0}^{1}, \ldots, \phi_{0}^{n+1}$.

Example 1. Let $\mathcal{N}_{1}^{+}\left(\mathbb{R}^{2, n+1}\right)$ and $\mathcal{N}_{1}\left(\mathbb{R}^{2, n+1}\right)$ denote, respectively, the manifolds of all oriented and unoriented isotropic lines through the origin in $\mathbb{R}^{2, n+1}$. For a nonzero isotropic vector $x \in \mathbb{R}^{2, n+1}$, we let $|[x]|$ and $[x]$ denote, respectively, the 
oriented and the unoriented line spanned by $x$. Consider the smooth maps

$$
\left\{\begin{array}{l}
\mathcal{E}^{1, n} \ni(\tau, \mathrm{y}) \longmapsto\left|\left[\left(\rho_{1}(\tau), \mathrm{y}\right)\right]\right| \in \mathcal{N}_{1}^{+}\left(\mathbb{R}^{2, n+1}\right), \\
\mathcal{E}^{1, n} \ni(\tau, \mathrm{y}) \longmapsto\left[\left(\rho_{1}(\tau), \mathrm{y}\right)\right] \in \mathcal{N}_{1}\left(\mathbb{R}^{2, n+1}\right),
\end{array}\right.
$$

where $\rho_{1}(\tau)={ }^{t}(\cos \tau, \sin \tau)$. The first map is invariant under the action of $\mathcal{T}_{2 \pi}$, while the second is invariant under the action of $\mathcal{T}_{\pi}^{\prime}$. They induce smooth diffeomorphisms

$$
\widehat{\eta}_{\mathrm{I}}: \mathcal{E}_{\mathrm{I}}^{1, n} \rightarrow \mathcal{N}_{1}^{+}\left(\mathbb{R}^{2, n+1}\right), \quad \widehat{\eta}_{\mathrm{II}}: \mathcal{E}_{\mathrm{II}}^{1, n} \rightarrow \mathcal{N}_{1}\left(\mathbb{R}^{2, n+1}\right) .
$$

Since for $n$ even, $\mathcal{E}_{\mathrm{II}}^{1, n}$ is not orientable, we will only consider $\mathcal{E}_{\mathrm{II}}^{1, n}$ when $n$ is odd. With the above identifications, we get smooth left actions of $\mathrm{O}_{+}^{\uparrow}(2, n+1)$ and $\mathrm{PO}_{+}^{\uparrow}(2, n+1):=\mathrm{O}_{+}^{\uparrow}(2, n+1) /\{ \pm I\}$ on the standard compact forms. These actions are conformal and preserve orientation and time-orientation. For dimensional reasons, $\mathrm{O}_{+}^{\uparrow}(2, n+1)$ and $\mathrm{PO}_{+}^{\uparrow}(2, n+1)$ are isomorphic to the restricted conformal groups of $\mathcal{E}_{\mathrm{I}}^{1, n}$ and $\mathcal{E}_{\mathrm{II}}^{1, n}$, respectively. Notice that the Maurer-Cartan forms of the groups coincide with the normal conformal connection forms.

\section{The Canonical COVERING OF $\mathrm{O}_{+}^{\uparrow}(2, n+1)$}

In this section we will build a nontrivial central extension $\widehat{\mathrm{O}}_{+}^{\uparrow}(2, n+1)$ of the pseudo-orthogonal group $\mathrm{O}_{+}^{\uparrow}(2, n+1)$ and will describe its center $\widehat{Z}(2, n+1)$.

\subsection{Transitive action of $\mathrm{O}_{+}^{\uparrow}(2, n+1)$ on the classical domain of type IV.}

Definition 6. Let $\Omega_{\mathrm{IV}}$ denote the set of $(n+1) \times 2$ real matrices defined by

$$
\Omega_{\mathrm{IV}}:=\left\{\beta \in \mathbb{R}(n+1,2) \mid I_{2}-{ }^{t} \beta \beta>0 \text {, i.e., } I_{2}-{ }^{t} \beta \beta \text { is positive definite }\right\} .
$$

The set $\Omega_{\mathrm{IV}}$ can be thought of as the open domain of $\mathbb{R}(n+1,2)$ consisting of all $(n+1) \times 2$ matrices $\beta=(\mathrm{uv})$ whose column vectors $\mathrm{u}, \mathrm{v} \in \mathbb{R}^{n+1}$ satisfy

$$
\|\mathrm{u}\|<1, \quad\|\mathrm{v}\|<1, \quad \mu(\mathrm{u}, \mathrm{v}):=\|\mathrm{u}\|^{2}+\|\mathrm{v}\|^{2}+(\mathrm{u} \cdot \mathrm{v})^{2}-\|\mathrm{u}\|^{2}\|\mathrm{v}\|^{2}<1,
$$

that is,

$$
\Omega_{\mathrm{IV}}=\left\{\beta=(\mathrm{uv}) \in \mathbb{R}(n+1,2) \mid\|\mathrm{u}\|^{2}<1,\|\mathrm{v}\|^{2}<1, \mu(\mathrm{u}, \mathrm{v})<1\right\} .
$$

Remark 4. Let $\mathfrak{D}$ denote the bounded domain of $\mathbb{C}^{n+1}$ defined by

$$
\mathfrak{D}:=\left\{\left.z \in \mathbb{C}^{n+1}\left|2^{t} z \bar{z}<1+\right|{ }^{t} z z\right|^{2}<2\right\} .
$$

The domain $\mathfrak{D}$ is known in the literature as the (complex) Lie ball. If $n=0$, it is the unit disk, $\mathbb{D}$, and if $n=1, \mathfrak{D} \cong \mathbb{D} \times \mathbb{D}$. According to Hua [18, §13], the Lie ball $\mathfrak{D}$ can be identified with the matrix domain $\Omega_{\mathrm{IV}}$. In fact, the mapping $H: \mathfrak{D} \rightarrow \Omega_{\mathrm{IV}}$, defined by

$$
H(z):=2\left(\begin{array}{ll}
z & \bar{z}
\end{array}\right)\left(\begin{array}{cc}
t_{z z+1} & \overline{t_{z z}}+1 \\
i\left({ }^{\left.t_{z z}-1\right)}\right. & -i\left(\overline{t_{z z}}-1\right)
\end{array}\right)^{-1}
$$

is a diffeomorphism of $\mathfrak{D}$ onto $\Omega_{\mathrm{IV}}$. Actually, if one identifies the matrix $\beta=(\mathrm{uv})$ with the vector $\mathrm{u}+i \mathrm{v} \in \mathbb{C}^{n+1}$, in order to obtain an almost complex structure on $\Omega_{\mathrm{IV}}$, the map $H$ becomes a holomorphic diffeomorphism. The domain $\mathfrak{D}(n \geq 2)$ is a classical irreducible bounded symmetric domain of type IV (cf. [7, 10, 17, 18, 19] for more details).

${ }^{7}$ For $\mathrm{u}, \mathrm{v} \in \mathbb{R}^{n+1}, \mathrm{u} \cdot \mathrm{v}$ denotes the usual dot product and $\|\mathrm{u}\|$ the corresponding norm. 
Notation 1. Let $\mathbf{X} \in \mathrm{O}_{+}^{\uparrow}(2, n+1)$ be written in block form as

$$
\mathbf{X}=\left(\begin{array}{ll}
\mathfrak{a}(\mathbf{X}) & \mathfrak{b}(\mathbf{X}) \\
\mathfrak{c}(\mathbf{X}) & \mathfrak{d}(\mathbf{X})
\end{array}\right)
$$

where $\mathfrak{a}(\mathbf{X}) \in \mathbb{R}(2,2), \mathfrak{b}(\mathbf{X}) \in \mathbb{R}(2, n+1), \mathfrak{c}(\mathbf{X}) \in \mathbb{R}(n+1,2), \mathfrak{d}(\mathbf{X}) \in \mathbb{R}(n+1, n+1)$ and

$$
\left(\begin{array}{ll}
\mathfrak{a}(\mathbf{X}) & \mathfrak{b}(\mathbf{X}) \\
\mathfrak{c}(\mathbf{X}) & \mathfrak{d}(\mathbf{X})
\end{array}\right)\left(\begin{array}{cc}
I_{2} & 0 \\
0 & -I_{n+1}
\end{array}\right)\left(\begin{array}{cc}
{ }^{t} \mathfrak{a}(\mathbf{X}) & { }^{t} \mathfrak{c}(\mathbf{X}) \\
t_{\mathfrak{b}}(\mathbf{X}) & \mathfrak{t}_{\mathfrak{d}(\mathbf{X})}
\end{array}\right)=\left(\begin{array}{cc}
I_{2} & 0 \\
0 & -I_{n+1}
\end{array}\right)
$$

that is,

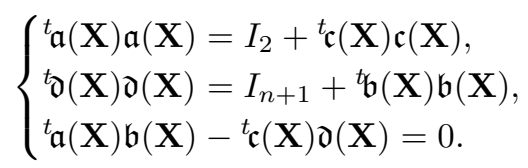

Since $\mathbf{X}$ preserves time and space-orientation, the timelike part $\mathfrak{a}(\mathbf{X})$ is invertible with $\operatorname{det} \mathfrak{a}(\mathbf{X})>0$. Similarly, the spacelike part $\mathfrak{d}(\mathbf{X})$ is invertible and $\operatorname{det} \mathfrak{d}(\mathbf{X})>0$.

With the above notation, it is easy to show that the group $\mathrm{O}_{+}^{\uparrow}(2, n+1)$ acts transitively on $\Omega_{\mathrm{IV}}$ by

$$
L_{\mathbf{X}}(\beta):=(\mathfrak{d}(\mathbf{X}) \beta+\mathfrak{c}(\mathbf{X}))(\mathfrak{b}(\mathbf{X}) \beta+\mathfrak{a}(\mathbf{X}))^{-1} .
$$

For a proof of this fact we refer to [18, §12]. The isotropy group at the origin $\mathrm{O}_{\mathrm{IV}}:=0_{(n+1) \times 2} \in \Omega_{\mathrm{IV}}$ is

$$
\mathrm{SO}(2) \times \mathrm{SO}(n+1) \cong\left\{\mathrm{S}(\mathrm{r}, \mathrm{R})=\left(\begin{array}{cc}
\mathrm{r} & 0 \\
0 & \mathrm{R}
\end{array}\right) \mid \mathrm{r} \in \mathrm{SO}(2), \mathrm{R} \in \mathrm{SO}(n+1)\right\} .
$$

This gives a coset expression for $\Omega_{\mathrm{IV}}$,

$$
\Omega_{\mathrm{IV}}=\mathrm{O}_{+}^{\uparrow}(2, n+1) / \mathrm{SO}(2) \times \mathrm{SO}(n+1) .
$$

The canonical projection of $\mathrm{O}_{+}^{\uparrow}(2, n+1)$ onto $\Omega_{\mathrm{IV}}$,

$$
\pi_{2}^{-}: \mathrm{O}_{+}^{\uparrow}(2, n+1) \rightarrow \Omega_{\mathrm{IV}}, \mathbf{X} \longmapsto L_{\mathbf{X}}\left(\mathrm{O}_{\mathrm{IV}}\right)=\mathfrak{c}(\mathbf{X}) \mathfrak{a}(\mathbf{X})^{-1},
$$

makes $\mathrm{O}_{+}^{\uparrow}(2, n+1)$ into a principal bundle over $\Omega_{\mathrm{IV}}$ with group $\mathrm{SO}(2) \times \mathrm{SO}(n+1)$.

The following is a well-known property of classical domains [17, 19. For future use, we state it explicitly for $\Omega_{\mathrm{IV}}$.

Lemma 4. The domain $\Omega_{\mathrm{IV}}$ is star-shaped with respect to the origin $\mathrm{O}_{\mathrm{IV}}=$ $0_{(n+1) \times 2}$. In particular, $\Omega_{\mathrm{IV}}$ is contractible.

Remark 5 . The above property of $\Omega_{\mathrm{IV}}$ also follows from the general approach to irreducible bounded symmetric domains based on the theory of Riemannian symmetric spaces. In fact, the domain $\Omega_{\mathrm{IV}}$ may be given the structure of a Riemannian symmetric space of noncompact type. As such, it has non-positive sectional curvature and negative definite Ricci tensor, which implies that $\Omega_{\mathrm{IV}}$ is simply connected, and hence diffeomorphic to Euclidean space $\mathbb{R}^{2(n+1)}$ (cf. [17, 27]).

Definition 7. Let $\mathrm{G}_{2}^{-}\left(\mathbb{R}^{2, n+1}\right)$ denote the Grassmannian of negative 2-planes, i.e., the Grassmannian of 2-dimensional subspaces $\mathbb{V}$ of $\mathbb{R}^{2, n+1}$ on which the scalar product $\langle$,$\rangle is negative definite.$ 
The matrix domain $\Omega_{\mathrm{IV}}$ can be identified with $\mathrm{G}_{2}^{-}\left(\mathbb{R}^{2, n+1}\right)$ by the mapping

$$
\mathbf{j}: \Omega_{\mathrm{IV}} \rightarrow \mathrm{G}_{2}^{-}\left(\mathbb{R}^{2, n+1}\right), \quad \mathbf{j}(\beta):=\operatorname{span}\left\{\mathbf{j}_{1}(\beta), \mathbf{j}_{2}(\beta)\right\},
$$

where, for each $\beta=(\mathrm{uv}) \in \Omega_{\mathrm{IV}}, \mathbf{j}_{1}(\beta)={ }^{t}\left(1,0,{ }^{t} \mathrm{u}\right)$ and $\mathbf{j}_{2}(\beta)={ }^{t}\left(0,1,{ }^{t} \mathrm{v}\right)$. In fact, we have the following.

Lemma 5. The mapping $\mathbf{j}: \Omega_{\mathrm{IV}} \rightarrow \mathrm{G}_{2}^{-}\left(\mathbb{R}^{2, n+1}\right)$ is a smooth diffeomorphism.

Proof. By construction, $\mathbf{j}$ is injective, differentiable and of maximal rank. Thus it suffices to show that $\mathbf{j}$ is surjective. Let $\mathbb{V} \in \mathrm{G}_{2}^{-}\left(\mathbb{R}^{2, n+1}\right)$ be a negative 2-plane and let $\left(\mathrm{v}_{1}, \mathrm{v}_{2}\right)$ be an orthogonal basis of $\mathbb{V}$. Let us write ${ }^{t} \mathrm{v}_{1}=\left({ }^{t} \mathrm{x},{ }^{t} \mathrm{y}\right)$ and ${ }^{t} \mathrm{v}_{2}=\left({ }^{t} \mathrm{x}^{\prime},{ }^{t} \mathrm{y}^{\prime}\right)$, where $\mathrm{x}, \mathrm{x}^{\prime} \in \mathbb{R}^{2}$ and $\mathrm{y}, \mathrm{y}^{\prime} \in \mathbb{R}^{n+1}$. The vectors $\mathrm{x}, \mathrm{x}^{\prime}$ and $\mathrm{y}, \mathrm{y}^{\prime}$ satisfy the identities

$$
-\|\mathrm{x}\|^{2}+\|\mathrm{y}\|^{2}=-\left\|\mathrm{x}^{\prime}\right\|^{2}+\left\|\mathrm{y}^{\prime}\right\|^{2}=-1, \quad-\mathrm{x} \cdot \mathrm{x}^{\prime}+\mathrm{y} \cdot \mathrm{y}^{\prime}=0 .
$$

First, observe that $\mathrm{x}$ and $\mathrm{x}^{\prime}$ must be different from zero. In fact, if $\mathrm{x}$ or $\mathrm{x}^{\prime}$ were zero, the first equation in (3.2) would imply $0 \leq\|y\|=-1$ or $0 \leq\left\|y^{\prime}\right\|=-1$, which is a contradiction. Next, we claim that $\mathrm{x}$ and $\mathrm{x}^{\prime}$ are linearly independent. Seeking a contradiction, suppose that $\mathrm{x}^{\prime}=t \mathrm{x}$, for a nonzero real number $t$. If we write $\mathrm{y}^{\prime} \cdot \mathrm{y}=\left\|\mathrm{y}^{\prime}\right\|\|\mathrm{y}\| \cos \theta$, where $\theta \in[0, \pi]$, the identities (3.2) can be rewritten as

$$
\|\mathrm{y}\|^{2}=\|\mathrm{x}\|^{2}-1, \quad\left\|\mathrm{y}^{\prime}\right\|^{2}=t^{2}\|\mathrm{x}\|^{2}-1, \quad t\|\mathrm{x}\|^{2}=\|\mathrm{y}\|\left\|\mathrm{y}^{\prime}\right\| \cos \theta,
$$

which implies

$$
\begin{aligned}
0 & =t^{2}\|\mathrm{x}\|^{4}-\|\mathrm{y}\|^{2}\left\|\mathrm{y}^{\prime}\right\|^{2} \cos ^{2} \theta \\
& =t^{2}\|\mathrm{x}\|^{4}-\left(\|\mathrm{x}\|^{2}-1\right)\left(t^{2}\|\mathrm{x}\|^{2}-1\right) \cos ^{2} \theta \\
& =t^{2}\|\mathrm{x}\|^{4}\left(1-\cos ^{2} \theta\right)+\left(\|\mathrm{x}\|^{2}\left(1+t^{2}\right)-1\right) \cos ^{2} \theta .
\end{aligned}
$$

By the first condition in (3.3), it follows that $\|x\|>1$. Taking this into account, the previous equation implies $\|\mathrm{x}\|^{2}\left(1+t^{2}\right)-1=0$, which is the desired contradiction. Now, since $\mathrm{x}$ and $\mathrm{x}^{\prime}$ are linearly independent, the $2 \times 2$ matrix $\left(\mathrm{xx}^{\prime}\right)$ is invertible and $\beta=\left(\mathrm{y} \mathrm{y}^{\prime}\right)\left(\mathrm{xx}^{\prime}\right)^{-1}$ is an element of $\Omega_{\mathrm{IV}}$ such that $\mathbf{j}(\beta)=\mathbb{V}$.

3.2. Construction of the canonical covering. For each $\beta=(\mathrm{uv}) \in \Omega_{\mathrm{IV}}$, where $\mathrm{u}={ }^{t}\left(u^{1}, \ldots, u^{n+1}\right), \mathrm{v}={ }^{t}\left(v^{1}, \ldots, v^{n+1}\right)$, we let ${ }^{8}$

$$
\mathrm{B}_{j}(\beta):={ }^{t}\left(u_{j}, v_{j}, \delta_{j}^{1}, \ldots, \delta_{j}^{n+1}\right), \quad j=1, \ldots, n+1,
$$

and, as above,

$$
\mathbf{j}_{1}(\beta)={ }^{t}\left(1,0, u^{1}, \ldots, u^{n+1}\right), \quad \mathbf{j}_{2}(\beta)={ }^{t}\left(0,1, v^{1}, \ldots, v^{n+1}\right) .
$$

Then

$$
\mathrm{B}(\beta)=\left(\mathbf{j}_{1}(\beta), \mathbf{j}_{2}(\beta), \mathrm{B}_{1}(\beta), \ldots, \mathrm{B}_{n+1}(\beta)\right)
$$

is a positive basis of $\mathbb{R}^{2, n+1}$, such that

- $\mathbf{j}_{1}(\beta), \mathbf{j}_{2}(\beta)$ span the negative 2 -space $\mathbf{j}(\beta)$;

- $\mathrm{B}_{1}(\beta), \ldots, \mathrm{B}_{n+1}(\beta)$ span the positive $(n+1)$-space $\mathbf{j}(\beta)^{\perp}$ of $\mathbb{R}^{2, n+1}$.

Consequently, by the Gram-Schmidt process, there is a unique smooth map $\mathfrak{T}: \Omega_{\mathrm{IV}} \rightarrow \mathrm{T}^{+}(n+3)$ into the group of upper triangular $(n+3) \times(n+3)$ matrices with positive entries on the main diagonal such that, for each $\beta \in \Omega_{\mathrm{IV}}, \mathrm{P}(\beta):=\mathrm{B}(\beta) \mathfrak{T}(\beta)$ belongs to $\mathrm{O}_{+}^{\uparrow}(2, n+1)$. The map

$$
\mathrm{P}: \Omega_{\mathrm{IV}} \ni \beta \longmapsto \mathrm{P}(\beta) \in \mathrm{O}_{+}^{\uparrow}(2, n+1)
$$

\footnotetext{
${ }^{8}$ Here $\delta_{j}^{i}$ denotes the Kronecker symbol.
} 
is a smooth global cross section of $\pi_{2}^{-}: \mathrm{O}_{+}^{\uparrow}(2, n+1) \rightarrow \Omega_{\mathrm{IV}}$. Let

$$
\begin{aligned}
& \widehat{\mathfrak{a}}: \Omega_{\mathrm{IV}} \rightarrow \mathrm{GL}_{+}(2, \mathbb{R}), \quad \widehat{\mathfrak{d}}: \Omega_{\mathrm{IV}} \rightarrow \mathrm{GL}_{+}(n+1, \mathbb{R}), \\
& \widehat{\mathfrak{c}}: \Omega_{\mathrm{IV}} \rightarrow \mathbb{R}(n+1,2), \quad \widehat{\mathfrak{b}}: \Omega_{\mathrm{IV}} \rightarrow \mathbb{R}(2, n+1)
\end{aligned}
$$

be the smooth maps defined by

$$
\mathrm{P}(\beta)=\left(\begin{array}{ll}
\widehat{\mathfrak{a}}(\beta) & \widehat{\mathfrak{b}}(\beta) \\
\widehat{\mathfrak{c}}(\beta) & \widehat{\mathfrak{d}}(\beta)
\end{array}\right), \quad \forall \beta \in \Omega_{\mathrm{IV}}
$$

Moreover, for each $t \in \mathbb{R}$, let

$$
\rho(t)=\left(\rho_{1}(t), \rho_{2}(t)\right)=\left(\begin{array}{rr}
\cos t & -\sin t \\
\sin t & \cos t
\end{array}\right) \in \mathrm{SO}(2) .
$$

We have the following.

Lemma 6. The map $\widehat{\mathfrak{a}}: \Omega_{\mathrm{IV}} \rightarrow \mathrm{GL}_{+}(2, \mathbb{R})$ has the following invariance properties:

$$
\widehat{\mathfrak{a}}(\mathrm{R} \beta)=\widehat{\mathfrak{a}}(\beta), \quad \widehat{\mathfrak{a}}\left(\beta \mathrm{r}^{-1}\right)^{-1} \mathrm{r} \widehat{\mathfrak{a}}(\beta) \in \mathrm{SO}(2),
$$

for each $\beta \in \Omega_{\mathrm{IV}}, \mathrm{R} \in \mathrm{SO}(n+1)$, and $\mathrm{r} \in \mathrm{SO}(2)$. In addition, there exists a unique smooth map $\eta: \Omega_{\mathrm{IV}} \times \mathrm{SO}(2) \rightarrow \mathbb{R}$, such that

$$
\rho(\eta(\beta, \mathrm{r}))=\widehat{\mathfrak{a}}\left(\beta \mathrm{r}^{-1}\right)^{-1} \mathrm{r} \widehat{\mathfrak{a}}(\beta) \mathrm{r}^{-1}, \quad \eta\left(\mathrm{O}_{\mathrm{IV}}, I_{2}\right)=0,
$$

for each $\beta \in \Omega_{\mathrm{IV}}$ and $\mathrm{r} \in \mathrm{SO}(2)$.

Proof. From the definition of $\mathrm{P}: \Omega_{\mathrm{IV}} \rightarrow \mathrm{O}_{+}^{\uparrow}(2, n+1)$, it follows that

$$
\begin{aligned}
& \widehat{\mathfrak{a}}(\beta)=\frac{1}{\sqrt{1-\|\mathrm{u}\|^{2}}}\left(\begin{array}{cc}
1 & \frac{\mathrm{u} \cdot \mathrm{v}}{\sqrt{1-\mu(\mathrm{u}, \mathrm{v})}} \\
0 & \frac{1-\|\mathrm{u}\|^{2}}{\sqrt{1-\mu(\mathrm{u}, \mathrm{v})}}
\end{array}\right), \\
& \widehat{\mathfrak{c}}(\beta)=\left(\frac{\mathrm{u}}{\sqrt{1-\|\mathrm{u}\|^{2}}} \frac{\left(1-\|\mathrm{u}\|^{2}\right) \mathrm{v}+(\mathrm{u} \cdot \mathrm{v}) \mathrm{u}}{\sqrt{1-\|\mathrm{u}\|^{2}} \sqrt{1-\mu(\mathrm{u}, \mathrm{v})}}\right),
\end{aligned}
$$

for each $\beta=(\mathrm{uv}) \in \Omega_{\mathrm{IV}}$. This implies that $\widehat{\mathfrak{a}}(\mathrm{R} \beta)=\widehat{\mathfrak{a}}(\beta)$, for every $\mathrm{R} \in \mathrm{SO}(n+1)$. For $\mathrm{r} \in \mathrm{SO}(2)$ and $\beta \in \Omega_{\mathrm{IV}}$, we have

$$
\mathbf{S}\left(\mathrm{r}, I_{n+1}\right) \mathrm{P}(\beta)=\left(\begin{array}{cc}
\mathrm{r} \widehat{\mathfrak{a}}(\beta) & \mathrm{r} \widehat{\mathfrak{b}}(\beta) \\
\widehat{\mathfrak{c}}(\beta) & \widehat{\mathfrak{d}}(\beta)
\end{array}\right) .
$$

A direct computation taking into account (3.4) and (3.5) shows that

$$
\pi_{2}^{-}\left[\mathbf{S}\left(\mathrm{r}, I_{n+1}\right) \mathrm{P}(\beta)\right]=\widehat{\mathfrak{c}}(\beta) \widehat{\mathfrak{a}}(\beta)^{-1} \mathrm{r}^{-1}=\beta \mathrm{r}^{-1} .
$$

This implies that the left hand side of (3.6) belongs to the fiber $\left(\pi_{2}^{-}\right)^{-1}\left(\beta \mathrm{r}^{-1}\right)$. As a consequence, we can write

$$
\mathbf{S}\left(\mathrm{r}, I_{n+1}\right) \mathrm{P}(\beta)=\mathrm{P}\left(\beta \mathrm{r}^{-1}\right)\left(\begin{array}{cc}
\widehat{\mathrm{r}} & 0 \\
0 & \widehat{\mathrm{R}}
\end{array}\right),
$$

for $\widehat{\mathrm{r}} \in \mathrm{SO}(2)$ and $\widehat{\mathrm{R}} \in \mathrm{SO}(n+1)$. Combining (3.6) and (3.7), we conclude that

$$
\widehat{\mathfrak{a}}\left(\beta \mathrm{r}^{-1}\right)^{-1} \mathrm{r} \widehat{\mathfrak{a}}(\beta)=\widehat{\mathrm{r}} \in \mathrm{SO}(2) .
$$

As for the existence of $\eta$, consider the map

$$
\widehat{\eta}: \Omega_{\mathrm{IV}} \times \mathrm{SO}(2) \rightarrow \mathrm{SO}(2),(\beta, \mathrm{r}) \longmapsto \widehat{\mathfrak{a}}\left(\beta \mathrm{r}^{-1}\right)^{-1} \mathrm{r} \widehat{\mathfrak{a}}(\beta) \mathrm{r}^{-1} .
$$


To conclude the proof it suffices to show that $\widehat{\eta}$ is homotopic to a constant map. Since $\Omega_{\mathrm{IV}}$ is star-shaped with respect to the origin $\mathrm{O}_{\mathrm{IV}}$, we can define the smooth map

$$
v: \Omega_{\mathrm{IV}} \times \mathrm{SO}(2) \times \mathbb{R} \rightarrow \mathrm{SO}(2),(\beta, \mathrm{r}, t) \longmapsto \widehat{\eta}(t \beta, \mathrm{r}) .
$$

By construction, $v(\beta, \mathrm{r}, 1)=\widehat{\eta}(\beta, \mathrm{r})$ and $v(\beta, \mathrm{r}, 0)=I_{2}$. This shows that $v$ is a homotopy between the constant map $I_{2}$ and $\widehat{\eta}$, which proves the claim.

Let

- $\mathfrak{m}: \Omega_{\mathrm{IV}} \times \Omega_{\mathrm{IV}} \rightarrow \Omega_{\mathrm{IV}}$,

- $\mathfrak{r}: \Omega_{\mathrm{IV}} \times \Omega_{\mathrm{IV}} \rightarrow \mathrm{SO}(2)$, and

- $\mathfrak{R}: \Omega_{\mathrm{IV}} \times \Omega_{\mathrm{IV}} \rightarrow \mathrm{SO}(n+1)$

be the smooth maps defined by requiring that

$$
\mathrm{P}(\beta) \mathrm{P}\left(\beta^{\prime}\right)=\mathrm{P}\left(\mathfrak{m}\left(\beta, \beta^{\prime}\right)\right)\left(\begin{array}{cc}
\mathfrak{r}\left(\beta, \beta^{\prime}\right) & 0 \\
0 & \mathfrak{R}\left(\beta, \beta^{\prime}\right)
\end{array}\right), \quad \forall \beta, \beta^{\prime} \in \Omega_{\mathrm{IV}} .
$$

Since $\Omega_{\mathrm{IV}}$ is simply connected, there exists a unique map $\Theta: \Omega_{\mathrm{IV}} \times \Omega_{\mathrm{IV}} \rightarrow \mathbb{R}$, such that $\mathfrak{r}\left(\beta, \beta^{\prime}\right)=\rho\left[\Theta\left(\beta, \beta^{\prime}\right)\right]$ and $\Theta\left(\mathrm{O}_{\mathrm{IV}}, \mathrm{O}_{\mathrm{IV}}\right)=0$. Let

- $\psi: \mathrm{O}_{+}^{\uparrow}(2, n+1) \rightarrow \mathrm{SO}(2)$,

- $\Psi: \mathrm{O}_{+}^{\uparrow}(2, n+1) \rightarrow \mathrm{SO}(n+1)$, and

- $\zeta: \mathrm{O}_{+}^{\uparrow}(2, n+1) \times \mathrm{O}_{+}^{\uparrow}(2, n+1) \rightarrow \mathbb{R}$

be the maps defined by requiring that

$$
\mathbf{X}=\mathrm{P}\left(\pi_{2}^{-}(\mathbf{X})\right)\left(\begin{array}{cc}
\psi(\mathbf{X}) & 0 \\
0 & \Psi(\mathbf{X})
\end{array}\right)
$$

and

$$
\zeta\left(\mathbf{X}, \mathbf{X}^{\prime}\right)=\Theta\left(\pi_{2}^{-}(\mathbf{X}), \Psi(\mathbf{X}) \pi_{2}^{-}\left(\mathbf{X}^{\prime}\right) \psi(\mathbf{X})^{-1}\right)+\eta\left(\pi_{2}^{-}\left(\mathbf{X}^{\prime}\right), \psi(\mathbf{X})\right),
$$

for each $\mathbf{X}, \mathbf{X}^{\prime} \in \mathrm{O}_{+}^{\uparrow}(2, n+1)$.

We are now in a position to state the first main result of the paper.

Theorem A. The subset of $\mathrm{O}_{+}^{\uparrow}(2, n+1) \times \mathbb{R}$ given by

$$
\widehat{\mathrm{O}}_{+}^{\uparrow}(2, n+1)=\left\{(\mathbf{X}, \tau) \in \mathrm{O}_{+}^{\uparrow}(2, n+1) \times \mathbb{R} \mid \psi(\mathbf{X})=\rho(\tau)\right\}
$$

is a connected embedded submanifold diffeomorphic to $\Omega_{\mathrm{IV}} \times \mathbb{R} \times \mathrm{SO}(n+1)$. The multiplication

$$
(\mathbf{X}, \tau) \star\left(\mathbf{X}^{\prime}, \tau^{\prime}\right)=\left(\mathbf{X} \mathbf{X}^{\prime}, \tau+\tau^{\prime}+\zeta\left(\mathbf{X}, \mathbf{X}^{\prime}\right)\right)
$$

gives $\widehat{\mathrm{O}}_{+}^{\uparrow}(2, n+1)$ the structure of a Lie group with neutral element $\left(I_{n+3}, 0\right)$ and inverse $(\mathbf{X}, \tau)^{-1}=\left(\mathbf{X}^{-1},-\tau-\zeta\left(\mathbf{X}, \mathbf{X}^{-1}\right)\right)$. Moreover, the map

$$
\sigma: \widehat{\mathrm{O}}_{+}^{\uparrow}(2, n+1) \ni(\mathbf{X}, \tau) \longmapsto \mathbf{X} \in \mathrm{O}_{+}^{\uparrow}(2, n+1)
$$

is a covering homomorphism of Lie groups. If $\widehat{\mathrm{Z}}(2, n+1)$ denotes the center of $\widehat{\mathrm{O}}_{+}^{\uparrow}(2, n+1)$, then

(1) $\widehat{\mathrm{Z}}(2, n+1)=\{(I, 2 \pi k) \mid k \in \mathbb{Z}\} \cong \mathbb{Z}$, if $n$ is even;

(2) $\widehat{\mathrm{Z}}(2, n+1)=\left\{\left((-1)^{k} I, \pi k\right) \mid k \in \mathbb{Z}\right\} \cong \mathbb{Z}_{2} \times \mathbb{Z}$, if $n$ is odd.

The proof of Theorem A is organized in four lemmas.

Lemma A1. The subset $\widehat{\mathrm{O}}_{+}^{\uparrow}(2, n+1)$ is an embedded submanifold diffeomorphic to $\Omega_{\mathrm{IV}} \times \mathbb{R} \times \mathrm{SO}(n+1)$ and $\sigma$ is a covering map. 
Proof. Let $\left(\mathbf{X}_{*}, \tau_{*}\right)$ be an element of $\widehat{\mathrm{O}}_{+}^{\uparrow}(2, n+1)$ and $U \subset \mathrm{O}_{+}^{\uparrow}(2, n+2)$ be a contractible open neighborhood of $\mathbf{X}_{*}$. Then there exists a unique differentiable function $v: U \rightarrow \mathbb{R}$ such that $\psi(\mathbf{X})=\rho(v(\mathbf{X}))$, for every $\mathbf{X} \in U$, satisfying $v\left(\mathbf{X}_{*}\right)=\tau_{*}$. Choose $\epsilon \in(0, \pi)$ and let

$$
U^{\prime}=\left\{\mathbf{X} \in U \mid v(\mathbf{X}) \in\left(\tau_{*}-\epsilon, \tau_{*}+\epsilon\right)\right\} .
$$

Then, $\widetilde{U}^{\prime}=U^{\prime} \times\left(\tau_{*}-\epsilon, \tau_{*}+\epsilon\right) \subset \mathrm{O}_{+}^{\uparrow}(2, n+1) \times \mathbb{R}$ is an open neighborhood of $\left(\mathbf{X}_{*}, \tau_{*}\right)$ such that

$$
\widetilde{U}^{\prime} \cap \widehat{\mathrm{O}}_{+}^{\uparrow}(2, n+1)=\left\{(\mathbf{X}, \tau) \in \widetilde{U}^{\prime} \mid \tau=v(\mathbf{X})\right\} .
$$

Hence, $\widehat{\mathrm{O}}_{+}^{\uparrow}(2, n+1) \cap \widetilde{U}^{\prime}$ is the graph of the function $v: U^{\prime} \rightarrow \mathbb{R}$. This implies that $\widehat{\mathrm{O}}_{+}^{\uparrow}(2, n+1)$ is a submanifold of $\mathrm{O}_{+}^{\uparrow}(2, n+1) \times \mathbb{R}$. Clearly, the map

$$
\Omega_{\mathrm{IV}} \times \mathbb{R} \times \mathrm{SO}(n+1) \ni(\beta, \tau, \mathrm{R}) \longmapsto(\mathrm{P}(\beta) \mathbf{S}(\rho(\tau), \mathrm{R}), \tau) \in \widehat{\mathrm{O}}_{+}^{\uparrow}(2, n+1)
$$

is bijective and of maximal rank. Thus, it is a diffeomorphism of $\Omega_{\mathrm{IV}} \times \mathbb{R} \times \mathrm{SO}(n+1)$ onto $\widehat{\mathrm{O}}_{+}^{\uparrow}(2, n+1)$.

By construction, $\sigma$ is a smooth surjective submersion. So, to conclude the proof it suffices to prove that each $\mathbf{X}_{*} \in \mathrm{O}_{+}^{\uparrow}(2, n+1)$ has an open neighborhood which is evenly covered by $\sigma$. Choose $\tau_{*} \in \mathbb{R}$ such that $\rho\left(\tau_{*}\right)=\psi\left(\mathbf{X}_{*}\right)$ and let $U_{*} \subset$ $\mathrm{O}_{+}^{\uparrow}(2, n+1)$ be the open neighborhood

$$
U_{*}=\left\{\mathbf{X} \in \mathrm{O}_{+}^{\uparrow}(2, n+1) \mid \psi(\mathbf{X}) \neq \rho\left(\tau_{*}+\pi\right)\right\} .
$$

We now prove that $U_{*}$ is evenly covered. For each $k \in \mathbb{Z}$ we consider the open neighborhood of $\widehat{\mathrm{O}}_{+}^{\uparrow}(2, n+1)$ defined by

$$
\widehat{U}_{k}=\left\{(\mathbf{X}, \tau) \in \widehat{\mathrm{O}}_{+}^{\uparrow}(2, n+1) \mid \mathbf{X} \in U_{*}, \tau \in\left(\tau_{*}+2 \pi k-\pi, \tau_{*}+2 \pi k+\pi\right)\right\} .
$$

Obviously, $\widehat{U}_{k} \cap \widehat{U}_{k}=\emptyset$, for every $h, k \in \mathbb{Z}, h \neq k$. By construction, $\bigcup_{k \in \mathbb{Z}} \widehat{U}_{k} \subset$ $\sigma^{-1}\left(U_{*}\right)$. Let $\left(\mathbf{X}^{\prime}, \tau^{\prime}\right)$ be an element of $\sigma^{-1}\left(U_{*}\right)$. Then $\mathbf{X}^{\prime} \in U_{*}$ and $\rho\left(\tau^{\prime}\right)=$ $\psi\left(\mathbf{X}^{\prime}\right) \neq \rho\left(\tau_{*}+\pi\right)$. Hence, $\tau^{\prime} \neq \tau_{*}+\pi \bmod 2 \pi \mathbb{Z}$. Therefore, there exists a unique $k \in \mathbb{Z}$ such that $\tau^{\prime} \in\left(\tau_{*}-\pi+2 \pi k, \tau_{*}+\pi+2 \pi k\right)$. This implies that $\left(\mathbf{X}^{\prime}, \tau^{\prime}\right) \in \widehat{U}_{k}$. Consequently, $\bigcup_{k \in \mathbb{Z}} \widehat{U}_{k}$ and $\sigma^{-1}\left(U_{*}\right)$ coincide. Choose $k \in \mathbb{Z}$ and let $\sigma_{k}: \widehat{U}_{k} \rightarrow U_{*}$ be the restriction of the map $\sigma$ to $\widehat{U}_{k}$. Then, $\sigma_{k}$ is a differentiable bijection of maximal rank and hence is a homeomorphism. This concludes the proof.

Lemma A2. The maps $\psi, \zeta$, and $\rho$ are related by

$$
\psi\left(\mathbf{X} \mathbf{X}^{\prime}\right)=\psi(\mathbf{X}) \psi\left(\mathbf{X}^{\prime}\right) \rho\left(\zeta\left(\mathbf{X}, \mathbf{X}^{\prime}\right)\right), \quad \forall \mathbf{X}, \mathbf{X}^{\prime} \in \mathrm{O}_{+}^{\uparrow}(2, n+1) .
$$

Proof. First we show that, for each $\beta \in \Omega_{\mathrm{IV}}$ and $\mathbf{S}(\mathrm{r}, \mathrm{R}) \in \mathrm{SO}(2) \times \mathrm{SO}(n+1)$,

$$
\psi(\mathbf{S}(\mathrm{r}, \mathrm{R}) \mathrm{P}(\beta))=\rho(\eta(\beta, \mathrm{r})) \mathrm{r} .
$$

By Lemma 6, $\widehat{\mathfrak{a}}(\mathrm{R} \beta)=\widehat{\mathfrak{a}}(\beta)$, for each $\beta \in \Omega_{\mathrm{IV}}$ and $\mathrm{R} \in \mathrm{SO}(n+1)$. Arguing as in the proof of Lemma 6 , from

$$
\mathbf{S}(\mathrm{r}, \mathrm{R}) \mathrm{P}(\beta)=\left(\begin{array}{ll}
\mathrm{r} \widehat{\mathfrak{a}}(\beta) & \mathrm{r} \widehat{\mathfrak{b}}(\beta) \\
\mathrm{R} \widehat{\mathfrak{c}}(\beta) & \mathrm{R} \widehat{\mathfrak{d}}(\beta)
\end{array}\right)
$$

it follows that $\mathbf{S}(\mathrm{r}, \mathrm{R}) \mathrm{P}(\beta) \in\left(\pi_{2}^{-}\right)^{-1}\left(\mathrm{R} \beta \mathrm{r}^{-1}\right)$. Therefore, we can write

$$
\mathbf{S}(\mathrm{r}, \mathrm{R}) \mathrm{P}(\beta)=\mathrm{P}\left(\mathrm{R} \beta \mathrm{r}^{-1}\right)\left(\begin{array}{cc}
\psi(\mathbf{S}(\mathrm{r}, \mathrm{R}) \mathrm{P}(\beta)) & 0 \\
0 & \Psi(\mathbf{S}(\mathrm{r}, \mathrm{R}) \mathrm{P}(\beta))
\end{array}\right) .
$$


By Lemma 6, comparing (3.9) and (3.10), we obtain

$$
\begin{aligned}
\psi(\mathbf{S}(\mathrm{r}, \mathrm{R}) \mathrm{P}(\beta)) & \left.=\widehat{\mathfrak{a}}\left(\mathrm{R} \beta \mathrm{r}^{-1}\right)^{-1} \mathrm{r} \widehat{\mathfrak{a}}(\beta)\right) \\
& =\widehat{\mathfrak{a}}\left(\beta \mathrm{r}^{-1}\right)^{-1} \mathrm{r} \widehat{\mathfrak{a}}(\beta) \\
& =\rho(\eta(\beta, \mathrm{r})) \mathrm{r} .
\end{aligned}
$$

Let $\mathbf{X}$ and $\mathbf{X}^{\prime}$ be two elements of $\mathrm{O}_{+}^{\uparrow}(2, n+1)$ and write

$$
\mathbf{X}=\mathrm{P}(\beta)\left(\begin{array}{cc}
\psi(\mathbf{X}) & 0 \\
0 & \Psi(\mathbf{X})
\end{array}\right), \quad \mathbf{X}^{\prime}=\mathrm{P}\left(\beta^{\prime}\right)\left(\begin{array}{cc}
\psi\left(\mathbf{X}^{\prime}\right) & 0 \\
0 & \Psi\left(\mathbf{X}^{\prime}\right)
\end{array}\right)
$$

where $\beta=\pi_{2}^{-}(\mathbf{X})$ and $\beta^{\prime}=\pi_{2}^{-}\left(\mathbf{X}^{\prime}\right)$. By (3.8) and (3.10), we obtain

$$
\begin{aligned}
& \mathbf{X} \mathbf{X}^{\prime}=\mathrm{P}(\beta)\left(\begin{array}{cc}
\psi(\mathbf{X}) & 0 \\
0 & \Psi(\mathbf{X})
\end{array}\right) \mathrm{P}\left(\beta^{\prime}\right)\left(\begin{array}{cc}
\psi\left(\mathbf{X}^{\prime}\right) & 0 \\
0 & \Psi\left(\mathbf{X}^{\prime}\right)
\end{array}\right) \\
& =\mathrm{P}(\beta) \mathrm{P}\left(\Psi(\mathbf{X}) \beta^{\prime} \psi(\mathbf{X})^{-1}\right)\left(\begin{array}{c}
\rho\left(\eta\left(\beta^{\prime}, \psi(\mathbf{X})\right)\right. \\
0
\end{array}(\mathbf{X}) \psi\left(\mathbf{X}^{\prime}\right) \underset{*}{0}\right) \\
& =\mathrm{P}\left(\mathfrak{m}\left(\beta, \Psi(\mathbf{X}) \beta^{\prime} \psi(\mathbf{X})^{-1}\right)\right)\left(\begin{array}{c}
\rho\left(\Theta\left(\beta, \Psi(\mathbf{X}) \beta^{\prime} \psi(\mathbf{X})^{-1}\right)+\eta\left(\beta^{\prime}, \psi(\mathbf{X})\right) \psi(\mathbf{X}) \psi\left(\mathbf{X}^{\prime}\right)\right. \\
0 \\
*
\end{array}\right) \\
& =\mathrm{P}\left(\mathfrak{m}\left(\beta, \Psi(\mathbf{X}) \beta^{\prime} \psi(\mathbf{X})^{-1}\right)\right)\left(\begin{array}{c}
\rho\left(\zeta\left(\mathbf{X}, \mathbf{X}^{\prime}\right)\right) \\
0
\end{array}(\mathbf{X}) \psi\left(\mathbf{X}^{\prime}\right) \underset{*}{0}\right) .
\end{aligned}
$$

This implies that

$$
\psi\left(\mathbf{X} \mathbf{X}^{\prime}\right)=\psi(\mathbf{X}) \psi\left(\mathbf{X}^{\prime}\right) \rho\left(\zeta\left(\mathbf{X}, \mathbf{X}^{\prime}\right)\right)
$$

as claimed.

Lemma A3. Let $\widehat{\mathrm{O}}_{+}^{\uparrow}(2, n+1)$ be defined as above. The multiplication

$$
(\mathbf{X}, \tau) \star\left(\mathbf{X}^{\prime}, \tau^{\prime}\right)=\left(\mathbf{X ~ X}^{\prime}, \tau+\tau^{\prime}+\zeta\left(\mathbf{X}, \mathbf{X}^{\prime}\right)\right)
$$

defines a Lie group structure on $\widehat{\mathrm{O}}_{+}^{\uparrow}(2, n+1)$ with neutral element $\mathbf{1}=\left(I_{n+3}, 0\right)$ and inverse $(\mathbf{X}, \tau)^{-1}=\left(\mathbf{X}^{-1},-\tau-\zeta\left(\mathbf{X}, \mathbf{X}^{-1}\right)\right)$.

Proof. First, we show that $\mathbf{1}=\left(I_{n+3}, 0\right)$ is a neutral element for the multiplication. By definition, $\mathbf{1} \star(\mathbf{X}, \tau) \in \sigma^{-1}(\mathbf{X})$, for each $(\mathbf{X}, \tau) \in \widehat{\mathrm{O}}_{+}^{\uparrow}(2, n+1)$. Then we can write $\mathbf{1} \star(\mathbf{X}, \tau)=(\mathbf{X}, \tau+2 \pi k(\mathbf{X}, \tau))$, where $k: \widehat{\mathrm{O}}_{+}^{\uparrow}(2, n+1) \rightarrow \mathbb{R}$ is a smooth map with integral values. Since $\widehat{\mathrm{O}}_{+}^{\uparrow}(2, n+1)$ is connected, $k$ is constant. By construction, $k(\mathbf{1})=0$. This implies that $\mathbf{1}$ is a left neutral element. The same reasoning shows that $\mathbf{1}$ is also a right neutral element. Next, we prove that each $(\mathbf{X}, \tau) \in \widehat{\mathrm{O}}_{+}^{\uparrow}(2, n+1)$ has a right inverse. Choose and fix $\left(\mathbf{X}^{-1}, \tau^{\prime}\right) \in \sigma^{-1}\left(\mathbf{X}^{-1}\right)$. Then

$$
(\mathbf{X}, \tau) \star\left(\mathbf{X}^{-1}, \tau^{\prime}\right)=\left(I_{n+3}, \tau+\tau^{\prime}+\zeta\left(\mathbf{X}, \mathbf{X}^{-1}\right)\right) \in \sigma^{-1}\left(I_{n+3}\right) .
$$

This implies that $\tau+\tau^{\prime}+\zeta\left(\mathbf{X}, \mathbf{X}^{-1}\right)=2 \pi m$, for some $m \in \mathbb{Z}$, from which it follows that $(\mathbf{X}, \tau) \star\left(\mathbf{X}^{-1}, \tau^{\prime}-2 \pi m\right)=\mathbf{1}$. We now prove that the right inverse is also a left inverse. Let $(\mathbf{X}, \tau) \in \widehat{\mathrm{O}}_{+}^{\uparrow}(2, n+1)$ and let $\left(\mathbf{X}^{-1}, \tau^{\prime}\right)$ be a right inverse of $(\mathbf{X}, \tau)$. We then have $\tau^{\prime}=-\tau-\zeta\left(\mathbf{X}, \mathbf{X}^{-1}\right)$, and hence

$$
\left(\mathbf{X}^{-1}, \tau^{\prime}\right) \star(\mathbf{X}, \tau)=\left(I_{n+3}, \zeta\left(\mathbf{X}^{-1}, \mathbf{X}\right)-\zeta\left(\mathbf{X}, \mathbf{X}^{-1}\right)\right) .
$$

This implies that the image of the smooth map

$$
\widetilde{\zeta}: \mathrm{O}_{+}^{\uparrow}(2, n+1) \ni \mathbf{X} \longmapsto \zeta\left(\mathbf{X}^{-1}, \mathbf{X}\right)-\zeta\left(\mathbf{X}, \mathbf{X}^{-1}\right) \in \mathbb{R}
$$


belongs to $2 \pi \mathbb{Z}$, from which it follows that $\widetilde{\zeta}$ is constant. Since $\widetilde{\zeta}\left(I_{n+3}\right)=0$, we conclude that $\widetilde{\zeta}$ vanishes identically. Thus $\left(\mathbf{X}^{-1}, \tau^{\prime}\right)$ is a left inverse and the map

$$
\widehat{\mathrm{O}}_{+}^{\uparrow}(2, n+1) \ni(\mathbf{X}, \tau) \longmapsto(\mathbf{X}, \tau)^{-1}=\left(\mathbf{X}^{-1},-\tau-\zeta\left(\mathbf{X}, \mathbf{X}^{-1}\right)\right) \in \widehat{\mathrm{O}}_{+}^{\uparrow}(2, n+1)
$$

is differentiable. We now prove that $\star$ is associative. Let $\left(\mathbf{X}^{\prime}, \tau^{\prime}\right)$ and $\left(\mathbf{X}^{\prime \prime}, \tau^{\prime \prime}\right)$ be two elements of $\widehat{\mathrm{O}}_{+}^{\uparrow}(2, n+1)$. Let $\Xi: \widehat{\mathrm{O}}_{+}^{\uparrow}(2, n+1) \rightarrow \widehat{\mathrm{O}}_{+}^{\uparrow}(2, n+1)$ be defined by

$$
\Xi(\mathbf{X}, \tau)=\left(\left(\left(\mathbf{X}^{\prime}, \tau^{\prime}\right) \star\left(\mathbf{X}^{\prime \prime}, \tau^{\prime \prime}\right)\right) \star(\mathbf{X}, \tau)\right) \star\left(\left(\mathbf{X}^{\prime}, \tau^{\prime}\right) \star\left(\left(\mathbf{X}^{\prime \prime}, \tau^{\prime \prime}\right) \star(\mathbf{X}, \tau)\right)\right)^{-1} .
$$

Since the multiplication of $\mathrm{O}_{+}^{\uparrow}(2, n+1)$ is associative, $\Xi(\mathbf{X}, \tau)$ belongs to $\sigma^{-1}\left(I_{n+3}\right)$. We can thus write $\Xi(\mathbf{X}, \tau)=\left(I_{n+3}, h(\mathbf{X}, \tau)\right)$, where $h: \widehat{\mathrm{O}}_{+}^{\uparrow}(2, n+1) \rightarrow \mathbb{R}$ is a smooth function taking values in $2 \pi \mathbb{Z}$. This implies that $h$ is constant. On the other hand, $h(\mathbf{1})=0$, which implies $\Xi=\mathbf{1}$, and hence the associativity of the product. This concludes the proof.

Lemma A4. Let $\widehat{\mathrm{Z}}(2, n+1)$ denote the center of $\widehat{\mathrm{O}}_{+}^{\uparrow}(2, n+1)$. Then,

- $\widehat{\mathrm{Z}}(2, n+1)=\{(I, 2 \pi k) \mid k \in \mathbb{Z}\}$, if $n$ is even;

- $\widehat{\mathrm{Z}}(2, n+1)=\left\{\left((-1)^{k} I, \pi k\right) \mid k \in \mathbb{Z}\right\}$, if $n$ is odd.

Proof. If $n$ is even, the center $\mathrm{Z}(2, n+1)$ of $\mathrm{O}_{+}^{\uparrow}(2, n+1)$ is trivial, while if $n$ is odd, the center is $\left\{ \pm I_{n+3}\right\}$. Thus, if $n$ is even, $\sigma^{-1}(\mathrm{Z}(2, n+1))=\{(I, 2 \pi k) \mid k \in \mathbb{Z}\}$, and if $n$ is odd, $\sigma^{-1}(\mathrm{Z}(2, n+1))=\left\{\left((-1)^{k} I, \pi k\right) \mid k \in \mathbb{Z}\right\}$. By construction, $\widehat{Z}(2, n+1) \subset$ $\sigma^{-1}(\mathrm{Z}(2, n+1))$. Suppose first that $n$ is even. Take $(I, 2 \pi k), k \in \mathbb{Z}$, and consider the smooth map

$$
f_{k}: \widehat{\mathrm{O}}_{+}^{\uparrow}(2, n+1) \ni(\mathbf{X}, \tau) \longmapsto(\mathbf{X}, \tau)^{-1} \star(I, 2 \pi k) \star(\mathbf{X}, \tau) \in \widehat{\mathrm{O}}_{+}^{\uparrow}(2, n+1) .
$$

Since $\sigma \circ f_{k}=I$, the image $\operatorname{Im}\left(f_{k}\right) \subset\{(I, 2 \pi m) \mid m \in \mathbb{Z}\}$, from which it follows that $f_{k}$ is constant. On the other hand, $f_{k}(I, 0)=(I, 2 \pi k)$, and hence $f_{k}(\mathbf{X}, \tau)=$ $(I, 2 \pi k)$. This implies that $(I, 2 \pi k)$ belongs to the center of $\widehat{\mathrm{O}}_{+}^{\uparrow}(2, n+1)$, which shows that $\widehat{Z}(2, n+1)=\{(I, 2 \pi k) \mid k \in \mathbb{Z}\}$, as claimed. Suppose now that $n$ is odd. Arguing as above, we can show that $\{(I, 2 \pi m) \mid m \in \mathbb{Z}\} \subset \widehat{\mathrm{Z}}(2, n+1)$. Next, take $(-I, \pi(1+2 k)), k \in \mathbb{Z}, k \neq 0$. Then the smooth map

$$
\widetilde{f}_{k}: \widehat{\mathrm{O}}_{+}^{\uparrow}(2, n+1) \ni(\mathrm{X}, \tau) \longmapsto(\mathbf{X}, \tau)^{-1} \star(-I, \pi(1+2 k)) \star(\mathbf{X}, \tau) \in \widehat{\mathrm{O}}_{+}^{\uparrow}(2, n+1)
$$

covers the constant map $-I$. Thus $\operatorname{Im}\left(\widetilde{f_{k}}\right) \subset\{(-I, \pi(1+2 m)) \mid m \in \mathbb{Z}\}$ and hence $\widetilde{f}_{k}$ is constant. On the other hand, $\widetilde{f}_{k}(I, 0)=(-I, \pi(1+2 k))$, which implies $\widetilde{f}_{k}(\mathbf{X}, \tau)=(-I, \pi(1+2 k))$, for every $(\mathbf{X}, \tau) \in \widehat{\mathrm{O}}_{+}^{\uparrow}(2, n+1)$. From this it follows that $\{(-I, \pi(1+2 k)) \mid k \in \mathbb{Z}\} \subset \widehat{\mathrm{Z}}(2, n+1)$.

The four lemmas combine to give the proof of Theorem

Remark 6. Let $\mathrm{s}: \operatorname{Spin}(2, n+1) \rightarrow \mathrm{O}_{+}^{\uparrow}(2, n+1)$ be the $2: 1$ spin covering group of $\mathrm{O}_{+}^{\uparrow}(2, n+1)$ (cf. [25). Then, the universal covering group of $\mathrm{O}_{+}^{\uparrow}(2, n+1)$ is the embedded submanifold of $\operatorname{Spin}(2, n+1) \times \mathbb{R}$ defined by

$$
\widehat{\mathrm{S}}(2, n+1)=\{(\mathbf{X}, \tau) \in \operatorname{Spin}(2, n+1) \times \mathbb{R} \mid \psi(s(\mathbf{X}))=\rho(\tau)\}
$$

with the multiplication $(\mathbf{X}, \tau) *\left(\mathbf{X}^{\prime}, \tau^{\prime}\right)=\left(\mathbf{X X}^{\prime}, \tau+\tau^{\prime}+\zeta\left(s(\mathbf{X}), s\left(\mathbf{X}^{\prime}\right)\right)\right)$. Note that $\widehat{\mathrm{S}}(2,3)$ is the universal covering group of $\operatorname{Sp}(4, \mathbb{R})$ while $\widehat{\mathrm{S}}(2,4)$ is the universal covering group of $\mathrm{SU}(2,2)$. In particular, this implies that $\widehat{\mathrm{O}}_{+}^{\uparrow}(2, n+1)$ cannot have a finite dimensional matrix representation (see, for instance, [29]). 


\section{LORENTZ MANIFOLDS WITH CONFORMAL GROUP OF MAXIMAL DIMENSION}

In this section, we prove that the central extension $\widehat{\mathrm{O}}_{+}^{\uparrow}(2, n+1)$ of $\mathrm{O}_{+}^{\uparrow}(2, n+1)$ is isomorphic to the restricted conformal group of the Einstein static universe and show that the integral compact forms of the first and second kind admit a restricted conformal transformation group of maximal dimension. Conversely, we prove that a conformal Lorentz manifold of dimension $n+1 \geq 3$ with restricted conformal group of maximal dimension is conformally equivalent to either the Einstein static universe, or to one of its integral compact forms.

\subsection{The restricted conformal group of $\mathcal{E}^{1, n}$ and its integral compact} forms.

Definition 8. Let define

- $\mathcal{T}_{h}=\left\{\left(I_{n+3}, 2 \pi h k\right) \mid k \in \mathbb{Z}\right\}$, for any integer $h \geq 1$,

- $\mathcal{T}_{h}^{\prime}=\left\{\left((-1)^{k} I_{n+3}, \pi(2 h+1) k\right) \mid k \in \mathbb{Z}\right\}$, for any integer $h \geq 0$ and $n$ odd.

Both $\mathcal{T}_{h}$ and $\mathcal{T}_{h}^{\prime}$ are discrete subgroups of the center $\widehat{\mathrm{Z}}(2, n+1)$ of $\widehat{\mathrm{O}}_{+}^{\uparrow}(2, n+1)$. Let

$$
\widehat{\mathrm{O}}_{+, h}^{\uparrow, \mathrm{I}}(2, n+1):=\widehat{\mathrm{O}}_{+}^{\uparrow}(2, n+1) / \mathcal{T}_{h}, \quad \widehat{\mathrm{O}}_{+, h}^{\uparrow, \mathrm{II}}(2, n+1):=\widehat{\mathrm{O}}_{+}^{\uparrow}(2, n+1) / \mathcal{T}_{h}^{\prime}
$$

be the corresponding quotient Lie groups.

We now state the second main result of the paper.

Theorem B. (1) The restricted conformal group of the Einstein static universe $\mathcal{E}^{1, n}$ is isomorphic to $\widehat{\mathrm{O}}_{+}^{\uparrow}(2, n+1)$.

(2) The restricted conformal group of the integral compact form of the first kind with index $h$ is isomorphic to $\widehat{\mathrm{O}}_{+, h}^{\uparrow, \mathrm{I}}(2, n+1)$.

(3) The restricted conformal group of the integral compact form of the second kind with index $h$ is isomorphic to $\widehat{\mathrm{O}}_{+, h}^{\uparrow, \mathrm{II}}(2, n+1)$.

Proof. Consider the left action L : $\mathrm{O}_{+}^{\uparrow}(2, n+1) \times \mathcal{E}_{\mathrm{I}}^{1, n} \rightarrow \mathcal{E}_{\mathrm{I}}^{1, n}$ of $\mathrm{O}_{+}^{\uparrow}(2, n+1)$ on the standard compact form of the first kind $\mathcal{E}_{\mathrm{I}}^{1, n}$ defined by

$$
\mathrm{L}_{\mathbf{X}}(\mathrm{x}, \mathrm{y})=\left(\frac{\mathfrak{a}(\mathbf{X}) \mathrm{x}+\mathfrak{b}(\mathbf{X}) \mathrm{y}}{\|\mathfrak{a}(\mathbf{X}) \mathrm{x}+\mathfrak{b}(\mathbf{X}) \mathrm{y}\|}, \frac{\mathfrak{c}(\mathbf{X}) \mathrm{x}+\mathfrak{d}(\mathbf{X}) \mathrm{y}}{\|\mathfrak{c}(\mathbf{X}) \mathrm{x}+\mathfrak{d}(\mathbf{X}) \mathrm{y}\|}\right) .
$$

The deck transformations of the covering $\pi_{\mathrm{I}, 1}: \mathcal{E}^{1, n} \rightarrow \mathcal{E}_{\mathrm{I}}^{1, n}$ are the translations

$$
\mathrm{T}_{k}: \mathcal{E}^{1, n} \ni(\tau, \mathrm{y}) \longmapsto(\tau+2 \pi k, \mathrm{y}) \in \mathcal{E}^{1, n}, \quad k \in \mathbb{Z}
$$

Let $\xi: \Omega_{\mathrm{IV}} \times \mathbb{R} \times \mathcal{E}^{1, n} \rightarrow \mathbb{S}^{1}$ be the smooth map defined by

$$
\xi(\beta, \vartheta,(\tau, \mathrm{y}))=\frac{\widehat{\mathfrak{a}}(\beta) \rho(\vartheta) \rho_{1}(\tau)+\widehat{\mathfrak{b}}(\beta) \mathrm{y}}{\left\|\widehat{\mathfrak{a}}(\beta) \rho(\vartheta) \rho_{1}(\tau)+\widehat{\mathfrak{b}}(\beta) \mathrm{y}\right\|} .
$$

Choose $O_{\mathcal{E}^{1, n}}={ }^{t}(0,(1,0, \ldots, 0)) \in \mathcal{E}^{1, n}$ as an origin. Since $\Omega_{\mathrm{IV}} \times \mathbb{R} \times \mathcal{E}^{1, n}$ is simply connected, there exists a unique smooth map $\widehat{\xi}: \Omega_{\mathrm{IV}} \times \mathbb{R} \times \mathcal{E}^{1, n} \rightarrow \mathbb{R}$, such that

$$
\rho(\widehat{\xi}(\beta, \vartheta,(\tau, \mathrm{y})))=\xi(\beta, \vartheta,(\tau, \mathrm{y})), \quad \widehat{\xi}\left(\mathrm{O}_{\mathrm{IV}}, 0, O_{\mathcal{E}^{1, n}}\right)=0,
$$

for every $(\beta, \vartheta,(\tau, \mathrm{y})) \in \Omega_{\mathrm{IV}} \times \mathbb{R} \times \mathcal{E}^{1, n}$. For $(\mathbf{X}, \vartheta) \in \widehat{\mathrm{O}}_{+}^{\uparrow}(2, n+1)$, consider the smooth map $\widehat{\mathrm{L}}_{(\mathbf{X}, \vartheta)}: \mathcal{E}^{1, n} \rightarrow \mathcal{E}^{1, n}$ given by

$$
\widehat{\mathrm{L}}_{(\mathbf{X}, \vartheta)}((\tau, \mathrm{y}))=\left(\widehat{\xi}\left(\pi_{2}^{-}(\mathbf{X}), \vartheta,(\tau, \Psi(\mathbf{X}) \mathrm{y})\right), \frac{\mathfrak{c}(\mathbf{X}) \rho_{1}(\tau)+\mathfrak{d}(\mathbf{X}) \mathrm{y}}{\left\|\mathfrak{c}(\mathbf{X}) \rho_{1}(\tau)+\mathfrak{d}(\mathbf{X}) \mathrm{y}\right\|}\right) .
$$


Let $\widehat{\mathrm{L}}: \widehat{\mathrm{O}}_{+}^{\uparrow}(2, n+1) \times \mathcal{E}^{1, n} \rightarrow \mathcal{E}^{1, n}$ be defined by

$$
\widehat{\mathrm{L}}((\mathbf{X}, \vartheta),(\tau, \mathrm{y}))=\widehat{\mathrm{L}}_{(\mathbf{X}, \vartheta)}((\tau, \mathrm{y})) \text {. }
$$

Then, $\widehat{\mathrm{L}}$ covers the action of $\mathrm{O}_{+}^{\uparrow}(2, n+1)$ on $\mathcal{E}_{\mathrm{I}}^{1, n}$, that is, $\pi_{\mathrm{I}, 1} \circ \widehat{\mathrm{L}}=\mathrm{L} \circ\left(\sigma, \pi_{\mathrm{I}, 1}\right)$. This implies that $\widehat{\mathrm{L}}_{(\mathbf{X}, \vartheta)}$ is an orientation and time-orientation preserving conformal transformation of $\mathcal{E}^{1, n}$.

To complete the proof of Theorem B we need the following.

Lemma B1. The map $\widehat{\mathrm{L}}$ defines an effective left action of $\widehat{\mathrm{O}}_{+}^{\uparrow}(2, n+1)$ on the Einstein static universe by restricted conformal transformations. Then, the restricted conformal group of $\mathcal{E}^{1, n}$ is isomorphic to $\widehat{\mathrm{O}}_{+}^{\uparrow}(2, n+1)$.

Proof of Lemma B1. First, note that $\widehat{\mathrm{L}}_{(I, 0)}=\operatorname{Id}_{\mathcal{E}^{1, n}}$. In fact, $\widehat{\mathrm{L}}_{(I, 0)}$ is a deck transformation of the covering $\pi_{\mathrm{I}, 1}$ and by definition and (4.2), $\widehat{\mathrm{L}}_{(I, 0)}\left(O_{\mathcal{E}^{1, n}}\right)=O_{\mathcal{E}^{1, n}}$. This implies that $\widehat{\mathrm{L}}_{(I, 0)}=\operatorname{Id}_{\mathcal{E}^{1, n}}$. We now prove that

$$
\widehat{\mathrm{L}}_{\left(\mathbf{X}_{*}, \vartheta_{*}\right)^{-1}} \circ \widehat{\mathrm{L}}_{\left(\mathbf{X}_{*}, \vartheta_{*}\right)}=\operatorname{Id}_{\mathcal{E}^{1, n}}, \quad \forall\left(\mathbf{X}_{*}, \vartheta_{*}\right) \in \widehat{\mathrm{O}}_{+}^{\uparrow}(2, n+1) .
$$

The composition $\widehat{\mathrm{L}}_{\left(\mathbf{X}_{*}, \vartheta_{*}\right)^{-1}} \circ \widehat{\mathrm{L}}_{\left(\mathbf{X}_{*}, \vartheta_{*}\right)}$ is a deck transformation of the covering $\pi_{\mathrm{I}, 1}$. Consider a smooth path $[0,1] \ni s \mapsto(\mathbf{X}(s), \vartheta(s)) \in \widehat{\mathrm{O}}_{+}^{\uparrow}(2, n+1)$, such that $(\mathbf{X}(0), \vartheta(0))=\left(\mathbf{X}_{*}, \vartheta_{*}\right)$ and $(\mathbf{X}(1), \vartheta(1))=(I, 0)$. Put $\lambda_{s}=\widehat{\mathrm{L}}_{(\mathbf{X}(s), \vartheta(s))^{-1} \circ}$ $\widehat{\mathrm{L}}_{(\mathbf{X}(s), \vartheta(s))}$ and consider the smooth map given by

$$
f:[0,1] \times \mathcal{E}^{1, n} \ni(s,(\tau, \mathrm{y})) \longmapsto f(s,(\tau, \mathrm{y}))=\lambda_{s}((\tau, \mathrm{y})) \in \mathcal{E}^{1, n} .
$$

By construction, $\lambda_{s}: \mathcal{E}^{1, n} \rightarrow \mathcal{E}^{1, n}$ is a deck transformation of the covering $\pi_{\mathrm{I}, 1}$, and hence $f(s,(\tau, \mathrm{y}))=(\tau+2 \pi k, \mathrm{y})$, where $k:[0,1] \rightarrow \mathbb{Z}, s \mapsto k(s)$, is independent of $s$. Since, by (4.2), $f\left(1, O_{\mathcal{E}^{1, n}}\right)=O_{\mathcal{E}^{1, n}}$, i.e., $\lambda_{1}\left(O_{\mathcal{E}^{1, n}}\right)=O_{\mathcal{E}^{1, n}}$, we have that $\lambda_{s}\left(O_{\mathcal{E}^{1, n}}\right)=O_{\mathcal{E}^{1, n}}$, and hence $\lambda_{s}=\operatorname{Id}_{\mathcal{E}^{1, n}}$ for every $s \in[0,1]$. In particular, $\lambda_{0}=\widehat{\mathrm{L}}_{\left(\mathbf{X}_{*}, \vartheta_{*}\right)^{-1}} \circ \widehat{\mathrm{L}}_{\left(\mathbf{X}_{*}, \vartheta_{*}\right)}=\operatorname{Id}_{\mathcal{E}^{1, n}}$, which implies that $\widehat{\mathrm{L}}_{\left(\mathbf{X}_{*}, \vartheta_{*}\right)}$ is a conformal diffeomorphism of $\mathcal{E}^{1, n}$ with inverse $\widehat{\mathrm{L}}_{\left(\mathbf{X}_{*}, \vartheta_{*}\right)^{-1}}$. We now show that

$$
\widehat{\mathrm{L}}_{\left(\mathbf{X}_{*}, \vartheta_{*}\right) \star\left(\mathbf{X}_{*}^{\prime}, \vartheta_{*}^{\prime}\right)}=\widehat{\mathrm{L}}_{\left(\mathbf{X}_{*}, \vartheta_{*}\right)} \circ \widehat{\mathrm{L}}_{\left(\mathbf{X}_{*}^{\prime}, \vartheta_{*}^{\prime}\right)},
$$

for every $\left(\mathbf{X}_{*}, \vartheta_{*}\right),\left(\mathbf{X}_{*}^{\prime}, \vartheta_{*}^{\prime}\right) \in \widehat{\mathrm{O}}_{+}^{\uparrow}(2, n+1)$. Let $\Phi_{\left(\left(\mathbf{X}_{*}, \vartheta_{*}\right),\left(\mathbf{X}_{*}^{\prime}, \vartheta_{*}^{\prime}\right)\right)}$ be the restricted conformal automorphism of $\mathcal{E}^{1, n}$ defined by

$$
\Phi_{\left(\left(\mathbf{X}_{*}, \vartheta_{*}\right),\left(\mathbf{X}_{*}^{\prime}, \vartheta_{*}^{\prime}\right)\right)}=\left(\widehat{\mathrm{L}}_{\left(\mathbf{X}_{*}, \vartheta_{*}\right) \star\left(\mathbf{X}_{*}^{\prime}, \vartheta_{*}^{\prime}\right)}\right)^{-1} \circ\left(\widehat{\mathrm{L}}_{\left(\mathbf{X}_{*}, \vartheta_{*}\right)} \circ \widehat{\mathrm{L}}_{\left(\mathbf{X}_{*}^{\prime}, \vartheta_{*}^{\prime}\right)}\right) .
$$

By definition, $\Phi_{\left(\left(\mathbf{X}_{*}, \vartheta_{*}\right),\left(\mathbf{X}_{*}^{\prime}, \vartheta_{*}^{\prime}\right)\right)}$ is a deck transformation of $\pi_{\mathrm{I}, 1}$. Next, consider the smooth paths $[0,1] \ni s \mapsto(\mathbf{X}(s), \vartheta(s)) \in \mathrm{O}_{+}^{\uparrow}(2, n+1)$ and $[0,1] \ni s \mapsto\left(\mathbf{X}^{\prime}(s), \vartheta^{\prime}(s)\right)$ $\in \mathrm{O}_{+}^{\uparrow}(2, n+1)$, such that $(\mathbf{X}(0), \vartheta(0))=\left(\mathbf{X}_{*}, \vartheta_{*}\right),(\mathbf{X}(1), \vartheta(1))=(I, 0)$ and $\left(\mathbf{X}^{\prime}(0), \vartheta^{\prime}(0)\right)=\left(\mathbf{X}_{*}^{\prime}, \vartheta_{*}^{\prime}\right),\left(\mathbf{X}^{\prime}(1), \vartheta^{\prime}(1)\right)=(I, 0)$, respectively. Consider the differentiable map

$$
\widetilde{f}:[0,1] \times \mathcal{E}^{1, n} \ni(s,(\tau, \mathrm{y})) \longmapsto \Phi_{\left((\mathbf{X}(s), \vartheta(s)),\left(\mathbf{X}^{\prime}(s), \vartheta^{\prime}(s)\right)\right)} \in \mathcal{E}^{1, n} .
$$

Then, for every $s \in[0,1]$, the map $\widetilde{f}_{s}: \mathcal{E}^{1, n} \ni(\tau, \mathrm{y}) \mapsto \widetilde{f}(s,(\tau, \mathrm{y})) \in \mathcal{E}^{1, n}$ is a deck transformation of $\pi_{\mathrm{I}, 1}$. Consequently, there exists $k \in \mathbb{Z}$, such that $\widetilde{f}(s,(\tau, \mathrm{y}))=$ 
$(\tau+2 \pi k, \mathrm{y})$. Since $\widetilde{f}_{1}=\operatorname{Id}_{\mathcal{E}^{1, n}}$, it follows that $\widetilde{f}_{s}=\operatorname{Id}_{\mathcal{E}^{1, n}}$, for every $s \in[0,1]$. This implies that

$$
\operatorname{Id}_{\mathcal{E}^{1, n}}=\widetilde{f}_{0}=\left(\widehat{\mathrm{L}}_{\left(\mathbf{X}_{*}, \vartheta_{*}\right) \star\left(\mathbf{X}_{*}^{\prime}, \vartheta_{*}^{\prime}\right)}\right)^{-1} \circ\left(\widehat{\mathrm{L}}_{\left(\mathbf{X}_{*}, \vartheta_{*}\right)} \circ \widehat{\mathrm{L}}_{\left(\mathbf{X}_{*}^{\prime}, \vartheta_{*}^{\prime}\right)}\right)
$$

We now prove that the action $\widehat{\mathrm{L}}$ is effective. Suppose $\widehat{\mathrm{L}}_{\left(\mathbf{X}_{*}, \vartheta_{*}\right)}=\operatorname{Id}_{\mathcal{E}^{1, n}}$. Since the action of $\mathrm{O}_{+}^{\uparrow}(2, n+1)$ on $\mathcal{E}_{\mathrm{I}}^{1, n}$ is effective, we have $\mathbf{X}_{*}=I_{n+3}$ and $\vartheta_{*}=2 \pi k$, where $k \in \mathbb{Z}$. Then, $\widehat{\mathrm{L}}_{\left(I_{n+3}, 2 \pi k\right)}(\tau, \mathrm{y})=(\tau+2 \pi k, \mathrm{y})$. Since $\widehat{\mathrm{L}}_{\left(\mathbf{X}_{*}, \vartheta_{*}\right)}=\operatorname{Id}_{\mathcal{E}^{1, n}}$, we must have $k=0$ and hence $\left(\mathbf{X}_{*}, \vartheta_{*}\right)=\left(I_{n+3}, 0\right)$. We have shown that $\widehat{\mathrm{O}}_{+}^{\uparrow}(2, n+1)$ is a connected Lie group acting effectively and transitively on $\mathcal{E}^{1, n}$ by restricted conformal transformations. Since $\widehat{\mathrm{O}}_{+}^{\uparrow}(2, n+1)$ has dimension $(n+3)(n+2) / 2$, it follows from Theorem 3 that $\widehat{\mathrm{O}}_{+}^{\uparrow}(2, n+1)$ is isomorphic to $\mathcal{C}_{+}^{\uparrow}\left(\mathcal{E}^{1, n}\right)$. This concludes the proof of Lemma $\mathrm{B}$.

We now resume the proof of Theorem B With reference to Definitions 112 and 8 , it is clear that $\mathcal{T}_{h}$ is the group of deck transformations of the covering $\pi_{\mathrm{I}, h}: \mathcal{E}^{1, n} \rightarrow \mathcal{E}_{\mathrm{I}, h}^{1, n}$, and similarly, if $n$ is odd, $\mathcal{T}_{h}^{\prime}$ is the group of deck transformations of the covering $\pi_{\mathrm{II}, h}: \mathcal{E}^{1, n} \rightarrow \mathcal{E}_{\mathrm{II}, h}^{1, n}$. The left action of $\widehat{\mathrm{O}}_{+}^{\uparrow}(2, n+1)$ on $\mathcal{E}^{1, n}$ descends to effective left actions by restricted conformal transformations $\mathrm{L}_{h}^{\mathrm{I}}: \widehat{\mathrm{O}}_{+, h}^{\uparrow, \mathrm{I}}(2, n+1) \times$ $\mathcal{E}_{\mathrm{I}, h}^{1, n} \rightarrow \mathcal{E}_{\mathrm{I}, h}^{1, n}$ and $\mathrm{L}_{h}^{\mathrm{II}}: \widehat{\mathrm{O}}_{+, h}^{\uparrow, \mathrm{II}}(2, n+1) \times \mathcal{E}_{\mathrm{II}, h}^{1, n} \rightarrow \mathcal{E}_{\mathrm{II}, h}^{1, n}$ on $\mathcal{E}_{\mathrm{I}, h}^{1, n}$ and $\mathcal{E}_{\mathrm{II}, h}^{1, n}$, respectively. Again by Theorem 3 , it follows that $\widehat{\mathrm{O}}_{+, h}^{\uparrow, \mathrm{I}}(2, n+1)$ and $\widehat{\mathrm{O}}_{+, h}^{\uparrow, \mathrm{II}}(2, n+1)$ are isomorphic to the restricted conformal groups of $\mathcal{E}_{\mathrm{I}, h}^{1, n}$ and $\mathcal{E}_{\mathrm{II}, h}^{1, n}$, respectively.

It is important to observe that any two integral compact forms with different indices belonging to the same series, as well as any two integral compact forms belonging to different series, cannot be conformally equivalent. More precisely, we have the following.

Proposition 7. (1) Any two integral compact forms $\mathcal{E}_{\mathrm{I}, k}^{1, n}$ and $\mathcal{E}_{\mathrm{I}, h}^{1, n}, k \neq h$, cannot be conformally diffeomorphic. (2) Any two integral compact forms $\mathcal{E}_{\mathrm{II}, k}^{1, n}$ and $\mathcal{E}_{\mathrm{II}, h}^{1, n}$, $k \neq h$, cannot be conformally diffeomorphic. (3) Any two integral compact forms $\mathcal{E}_{\mathrm{II}, k}^{1, n}$ and $\mathcal{E}_{\mathrm{I}, h}^{1, n}$ cannot be conformally diffeomorphic.

Proof. (1) Suppose $f: \mathcal{E}_{\mathrm{I}, k}^{1, n} \rightarrow \mathcal{E}_{\mathrm{I}, h}^{1, n}$ is a conformal diffeomorphism. Since $\mathcal{E}^{1, n}$ is simply connected, there exists an orientation preserving conformal transformation of maximal rank $\tilde{f}: \mathcal{E}^{1, n} \rightarrow \mathcal{E}^{1, n}$ that covers $f$. Let $\dot{\tilde{f}}: \widehat{\mathrm{O}}_{+}^{\uparrow}(2, n+1) \rightarrow \widehat{\mathrm{O}}_{+}^{\uparrow}(2, n+1)$ be the conformal prolongation of $\tilde{f}$. Since $\dot{\tilde{f}}$ preserves the Maurer-Cartan form of $\widehat{\mathrm{O}}_{+}^{\uparrow}(2, n+1)$, $\dot{\tilde{f}}$ coincides with the left multiplication by an element $(\mathbf{H}, \tau)$ of $\widehat{\mathrm{O}}_{+}^{\uparrow}(2, n+1)$. This implies that, for each $(t, \mathrm{x}) \in \mathcal{E}^{1, n}$,

$$
\tilde{f}((t, \mathrm{x}))=\widehat{\mathrm{L}}_{(\mathbf{H}, \tau)}((t, \mathrm{x})) .
$$

Since $\tilde{f}$ covers $f, \tilde{f}$ takes the fibers of the covering $\pi_{\mathrm{I}, k}: \mathcal{E}^{1, n} \rightarrow \mathcal{E}_{\mathrm{I}, k}^{1, n}$ to the fibers of the covering $\pi_{\mathrm{I}, h}: \mathcal{E}^{1, n} \rightarrow \mathcal{E}_{\mathrm{I}, h}^{1, n}$. In particular, if we set $(\mathfrak{t}, \mathrm{y}):=\widehat{\mathrm{L}}_{(\mathbf{H}, \tau)}((t, \mathrm{x}))$ and take into account that $\mathcal{T}_{h}$ (cf. Definition 8 ) is the deck transformation group of the covering $\pi_{\mathrm{I}, h}$, then

$$
\widehat{\mathrm{L}}_{(\mathbf{H}, \tau)}((t+2 \pi k, \mathrm{x}))=(\mathfrak{t}+2 \pi m h, \mathrm{y}), \quad m \in \mathbb{Z} .
$$


Using the fact that $(I, 2 \pi k)$ belongs to the center of $\widehat{\mathrm{O}}_{+}^{\uparrow}(2, n+1)$, the left-hand-side of (4.5) can be written as

$$
\begin{aligned}
\widehat{\mathrm{L}}_{(\mathbf{H}, \tau)}((t+2 \pi k, \mathrm{x})) & =\left(\widehat{\mathrm{L}}_{(\mathbf{H}, \tau)} \circ \widehat{\mathrm{L}}_{(I, 2 \pi k)}\right)((t, \mathrm{x})) \\
& =\widehat{\mathrm{L}}_{(I, 2 \pi k)}\left(\widehat{\mathrm{L}}_{(\mathbf{H}, \tau)}((t, \mathrm{x}))\right) \\
& =(\mathfrak{t}+2 \pi k, \mathrm{y}) .
\end{aligned}
$$

From (4.5) and (4.6), it follows that $k=m h, m \in \mathbb{Z}$. Since $h, k>0, m>0$. Repeating the argument for the inverse map $f^{-1}$ yields $h=n k, n \in \mathbb{Z}, n>0$. In conclusion, if $f$ is a conformal diffeorphism, then $k=h$.

(2) Suppose $f: \mathcal{E}_{\mathrm{II}, k}^{1, n} \rightarrow \mathcal{E}_{\mathrm{II}, h}^{1, n}$ is a conformal diffeomorphism. Let $\tilde{f}: \mathcal{E}^{1, n} \rightarrow$ $\mathcal{E}^{1, n}$ be the conformal transformation that covers $f$ and let $\dot{\tilde{f}}$ be the conformal prolongation of $\tilde{f}$. As above, $\dot{\tilde{f}}$ coincides with the left multiplication by an element $(\mathbf{H}, \tau)$ of $\widehat{\mathrm{O}}_{+}^{\uparrow}(2, n+1)$ and, for each $(t, \mathrm{x}) \in \mathcal{E}^{1, n}$,

$$
\tilde{f}((t, \mathrm{x}))=\widehat{\mathrm{L}}_{(\mathbf{H}, \tau)}((t, \mathrm{x})) .
$$

As $\tilde{f}$ covers $f, \tilde{f}$ takes the fibers of the covering $\pi_{\mathrm{II}, k}: \mathcal{E}^{1, n} \rightarrow \mathcal{E}_{\mathrm{II}, k}^{1, n}$ to the fibers of the covering $\pi_{\mathrm{II}, h}: \mathcal{E}^{1, n} \rightarrow \mathcal{E}_{\mathrm{II}, h}^{1, n}$. In particular, taking into account that $\mathcal{T}_{h}^{\prime}$ (cf. Definition (8) is the deck transformation group of $\pi_{\mathrm{II}, h}$, we have

$$
\widehat{\mathrm{L}}_{(\mathbf{H}, \tau)}((t+(2 k+1) \pi,-\mathrm{x}))=\left\{\begin{array}{l}
(\mathfrak{t}+\pi(2 h+1) 2 q, \mathrm{y}), \quad q \in \mathbb{Z}, \\
(\mathfrak{t}+\pi(2 h+1)(2 q+1),-\mathrm{y}), \quad q \in \mathbb{Z},
\end{array}\right.
$$

where again $(\mathfrak{t}, \mathrm{y}):=\widehat{\mathrm{L}}_{(\mathbf{H}, \tau)}((t, \mathrm{x}))$. Using the fact that $(-I,(2 k+1) \pi)$ belongs to the center of $\widehat{\mathrm{O}}_{+}^{\uparrow}(2, n+1)$, the left-hand-side of (4.7) can be written as

$$
\begin{aligned}
\widehat{\mathrm{L}}_{(\mathbf{H}, \tau)}((t+(2 k+1) \pi,-\mathrm{x})) & =\left(\widehat{\mathrm{L}}_{(\mathbf{H}, \tau)} \circ \widehat{\mathrm{L}}_{(-I,(2 k+1) \pi)}\right)((t, \mathrm{x})) \\
& =\widehat{\mathrm{L}}_{(-I,(2 k+1) \pi)}\left(\widehat{\mathrm{L}}_{(\mathbf{H}, \tau)}((t, \mathrm{x}))\right) \\
& =(\mathfrak{t}+(2 k+1) \pi,-\mathrm{y}) .
\end{aligned}
$$

From (4.7) and (4.8), it follows that $2 k+1=(2 q+1)(2 h+1), q \in \mathbb{Z}$. Since $h, k \geq 0,2 q+1>0$. Repeating the argument for the inverse map $f^{-1}$ yields $2 h+1=(2 s+1)(2 k+1), s \in \mathbb{Z}, 2 s+1>0$. Therefore, if $f$ is a conformal diffeomorphism, $k=h$.

(3) Seeking a contradiction, we suppose that $f: \mathcal{E}_{\mathrm{II}, k}^{1, n} \rightarrow \mathcal{E}_{\mathrm{I}, h}^{1, n}$ is a conformal diffeomorphism. Arguing as in the proof of points (1) and (2), we are led to

$$
(\mathfrak{t}+(2 k+1) \pi,-\mathrm{y})=(\mathfrak{t}+2 \pi m h, \mathrm{y}), \quad m \in \mathbb{Z},
$$

which is the desired contradiction.

4.2. Lorentz manifolds with restricted conformal group of maximal dimension. In this section, we prove the following result about Lorentz manifolds with restricted conformal group of maximal dimension.

Theorem C. Let $\mathbb{M}$ be a $(n+1)$-dimensional, $n \geq 2$, connected, oriented, timeoriented conformal Lorentz manifold such that

$$
\operatorname{dim}\left(\mathcal{C}_{+}^{\uparrow}(\mathbb{M})\right)=\frac{1}{2}(n+3)(n+2) .
$$


Then

(1) If $\mathbb{M}$ is simply connected, then $\mathbb{M}$ is conformally equivalent to the Einstein universe $\mathcal{E}^{1, n}$.

(2) If $\mathbb{M}$ is not simply connected and $n$ is even, then $\mathbb{M}$ is conformally equivalent to an integral compact form of the first kind of the Einstein universe.

(3) If $\mathbb{M}$ is not simply connected and $n$ is odd, then $\mathbb{M}$ is conformally equivalent to either an integral compact form of the first kind of the Einstein universe, or to an integral compact form of the second kind of the Einstein universe.

Proof. If $\operatorname{dim}\left(\mathcal{C}_{+}^{\uparrow}(\mathbb{M})\right)=\frac{1}{2}(n+3)(n+2)$, from Theorem 3 it follows that:

- the Cartan conformal bundle $\mathrm{Q}(\mathbb{M})$ is a Lie group acting effectively on $\mathbb{M}$ by restricted conformal transformations;

- the normal conformal connection of $\mathrm{Q}(\mathbb{M})$, denoted by $\phi^{\prime}$, coincides with the Maurer-Cartan form;

- Let $e^{\prime} \in \mathrm{Q}(\mathbb{M})$ be the neutral element and put $p_{*}^{\prime}=\pi_{\mathrm{Q}(\mathbb{M})}\left(e^{\prime}\right)$. The isotropy subgroup $\mathrm{H}^{\prime} \subset \mathrm{Q}(\mathbb{M})$ of the point $p_{*}^{\prime}$ coincides with the fiber $\pi_{\mathrm{Q}(\mathbb{M})}^{-1}\left(p_{*}^{\prime}\right)$, and hence it is isomorphic to $\mathrm{H}_{+}^{\uparrow}(2, n+1)$;

- $\mathrm{H}^{\prime}$ is the maximal integral submanifold through $e^{\prime}$ of the left-invariant completely integrable Pfaffian differential systems generated by the 1-forms $\phi_{0}^{\prime}$, $j=1, \ldots, n+1$.

Analogous conclusions hold for the Einstein static universe, namely:

- $\mathrm{Q}\left(\mathcal{E}^{1, n}\right) \cong \widehat{\mathrm{O}}_{+}^{\uparrow}(2, n+1)$ is a Lie group acting effectively on $\mathcal{E}^{1, n}$ by restricted conformal transformations;

- the normal conformal connection of $\mathrm{Q}\left(\mathcal{E}^{1, n}\right)$, denoted by $\phi^{\prime \prime}$, coincides with the Maurer-Cartan form;

- Let $e^{\prime \prime} \in \mathrm{Q}\left(\mathcal{E}^{1, n}\right)$ be the neutral element and put $p_{*}^{\prime \prime}=\pi_{\mathrm{Q}\left(\mathcal{E}^{1, n}\right)}\left(e^{\prime \prime}\right)$. The stabilizer of the point $p_{*}^{\prime \prime}$ coincides with the fiber $\pi_{\mathrm{Q}\left(\mathcal{E}^{1, n}\right)}^{-1}\left(p_{*}^{\prime \prime}\right)$. It is a closed Lie subgroup $\mathrm{H}^{\prime \prime} \subset \mathrm{Q}\left(\mathcal{E}^{1, n}\right)$, isomorphic to $\mathrm{H}_{+}^{\uparrow}(2, n+1)$.

- $\mathrm{H}^{\prime \prime}$ is the maximal integral sub-manifold through $e^{\prime \prime}$ of the left-invariant completely integrable Pfaffian differential systems generated by the 1 -forms $\phi_{0}^{\prime \prime}{ }_{0}^{j}, j=1, \ldots, n+1$.

For point (1), let $\widehat{\mathrm{S}}(2, n+1)$ be the universal covering group of $\mathrm{Q}\left(\mathcal{E}^{1, n}\right)$. Since $\mathbb{M}$ is connected and the fibers of $\mathrm{Q}(\mathbb{M})$ are connected, from the exact homotopy sequence of a principal bundle [32, in this case $\pi_{\mathrm{Q}}: \mathrm{Q}(\mathbb{M}) \rightarrow \mathbb{M}$, it follows that $\mathrm{Q}(\mathbb{M})$ is connected. Hence $\widehat{\mathrm{S}}(2, n+1)$ is also the universal covering group of $\mathrm{Q}(\mathbb{M})$. Let

$$
\mathrm{pr}_{1}: \widehat{\mathrm{S}}(2, n+1) \rightarrow \mathrm{Q}(\mathbb{M}), \quad \mathrm{pr}_{2}: \widehat{\mathrm{S}}(2, n+1) \rightarrow \mathrm{Q}\left(\mathcal{E}^{1, n}\right)
$$

be the corresponding covering homomorphisms. They can be chosen in a way that

$$
\phi=\operatorname{pr}_{1}^{*}\left(\phi^{\prime}\right)=\operatorname{pr}_{2}^{*}\left(\phi^{\prime \prime}\right),
$$

where $\phi$ is the Maurer-Cartan form of $\widehat{\mathrm{S}}(2, n+1)$. Let $e \in \widehat{\mathrm{S}}(2, n+1)$ be the multiplicative unit and $\widetilde{\mathrm{H}}$ be the maximal integral submanifold trough $e$ of the completely integrable Pfaffian differential system generated by $\phi_{0}^{1}, \ldots, \phi_{0}^{n+1}$. The universal covering $\widehat{\mathrm{S}}(2, n+1)$ acts almost effectively on $\mathbb{M}$ and $\mathcal{E}^{1, n}$. Let $\widetilde{\mathrm{H}}^{\prime}$ and $\widetilde{\mathrm{H}}^{\prime \prime}$ be the stabilizers of these actions at $p_{*}^{\prime}$ and $p_{*}^{\prime \prime}$, respectively. Since $\widehat{\mathrm{S}}(2, n+1)$, $\mathbb{M}$, and $\mathcal{E}^{1, n}$ are connected and simply connected, from the exact homotopy sequence of a 
principal bundle it follows that $\widetilde{\mathrm{H}}^{\prime}$ and $\widetilde{\mathrm{H}}^{\prime \prime}$ are connected. By construction, they are integral manifolds of the Pfaffian differential system $\phi_{0}^{1}=\cdots=\phi^{n+1}=0$. Hence $\widetilde{\mathrm{H}}^{\prime}=\widetilde{\mathrm{H}}^{\prime \prime}=\widetilde{\mathrm{H}}$. Therefore, $\mathbb{M}$ and $\mathcal{E}^{1, n}$ are both diffeomorphic to the homogeneous space $\widehat{\mathrm{S}}(2, n+1) / \widetilde{\mathrm{H}}$. Thus there exists a unique diffeomorphism $\Phi: \mathbb{M} \rightarrow \mathcal{E}^{1, n}$, such that $\Phi \circ \widehat{\pi}_{\mathbb{M}}=\widehat{\pi}_{\mathcal{E}^{1, n}}$, where

$$
\widehat{\pi}_{\mathbb{M}}: \widehat{\mathrm{S}}(2, n+1) \rightarrow \mathbb{M}, \quad \widehat{\pi}_{\mathcal{E}^{1, n}}: \widehat{\mathrm{S}}(2, n+1) \rightarrow \mathcal{E}^{1, n}
$$

are the two natural bundle maps. Now we show that $\Phi$ is an orientation and time-orientation preserving conformal map. We cover $\mathbb{M}$ with a family $\left\{U_{\alpha}\right\}_{\alpha \in \mathcal{C}^{\prime}}$ of simply connected open neighborhoods such that, for each $\alpha \in \mathcal{C}^{\prime}$, there exists a cross section $\dot{\mathcal{A}}_{\alpha}: U_{\alpha} \rightarrow \mathrm{Q}(\mathbb{M})$. For each $\alpha$, we choose a lift $\dot{\mathcal{A}}_{\alpha}^{\prime}$ of $\dot{\mathcal{A}}_{\alpha}$ to $\widehat{\mathrm{S}}(2, n+1)$. Then, the map

$$
\dot{\mathcal{B}}_{\alpha}:=\operatorname{pr}_{2} \circ \dot{\mathcal{A}}_{\alpha}^{\prime} \circ \Phi^{-1}: \Phi\left(U_{\alpha}\right) \rightarrow \mathrm{Q}\left(\mathcal{E}^{1, n}\right)
$$

is a cross section of $Q\left(\mathcal{E}^{1, n}\right)$. Put $\phi_{\alpha}=\dot{\mathcal{A}}_{\alpha}^{*}\left(\phi^{\prime}\right)$ and $\widetilde{\phi}_{\alpha}=\dot{\mathcal{B}}_{\alpha}^{*}\left(\phi^{\prime \prime}\right)$. Then, $\left(\Phi^{-1}\right)^{*}\left(\widetilde{\phi}_{\alpha}\right)$ $=\phi_{\alpha}$. Hence, $\left(\phi_{\alpha 0}^{1}, \ldots, \phi_{\alpha 0}^{n+1}\right)$ and $\left(\widetilde{\phi}_{\alpha, 0}^{1}, \ldots, \widetilde{\phi}_{\alpha, 0}^{n+1}\right)$ are two positive oriented and time-oriented conformal coframes, defined on the open neighborhoods $U_{\alpha} \subset \mathbb{M}$ and $\Phi\left(U_{\alpha}\right) \subset \mathcal{E}^{1, n}$, respectively, such that $\left(\Phi^{-1}\right)^{*}\left(\widetilde{\phi}_{\alpha, 0}^{i}\right)=\phi_{\alpha 0}^{i}, i=1, \ldots, n+1$. This implies that the restriction of $\Phi$ to $U_{\alpha}$ is an orientation and time-orientation preserving conformal diffeomorphism.

As for points (2) and (3), if $\mathbb{M}$ is not simply connected, let $\mathrm{p}_{\mathbb{M}}: \mathbb{M}^{*} \rightarrow \mathbb{M}$ be the universal covering space of $\mathbb{M}$, equipped with the oriented, time-oriented Lorentz structure such that $\mathrm{p}_{\mathbb{M}}$ is conformal, orientation and time-orientation preserving. The group $\Gamma$ of deck transformations of the covering $\mathrm{p}_{\mathbb{M}}$ is contained in the restricted conformal group $\mathcal{C}_{+}^{\uparrow}\left(\mathbb{M}^{*}\right)$ of $\mathbb{M}^{*}$. Then, according to [8, Theorem 9.1, page 63], there exists a covering group $\pi_{\mathcal{C}}: \mathrm{G} \rightarrow \mathcal{C}_{+}^{\uparrow}(\mathbb{M})$ and an effective action

$$
\widehat{\mathrm{L}}: \mathrm{G} \times \mathbb{M}^{*} \rightarrow \mathbb{M}^{*}
$$

such that $\mathrm{L}_{\pi_{\mathcal{C}}(g)} \circ \mathrm{p}_{\mathbb{M}}=\mathrm{p}_{\mathbb{M}} \circ \widehat{\mathrm{L}}_{g}$. This implies that $\mathrm{G}$ acts by orientation and time-orientation preserving conformal automorphisms. Thus, $\operatorname{dim}\left(\mathcal{C}_{+}^{\uparrow}\left(\mathbb{M}^{*}\right)\right)=(n+$ $3)(n+2) / 2$. By the first part of the proof, we may conclude that $\mathbb{M}^{*}$ can be identified with $\mathcal{E}^{1, n}$ and $\mathrm{G}$ with $\mathcal{C}_{+}^{\uparrow}\left(\mathcal{E}^{1, n}\right) \cong \widehat{\mathrm{O}}_{+}^{\uparrow}(2, n+1)$. In addition, the action of $\widehat{\mathrm{O}}_{+}^{\uparrow}(2, n+1)$ descends to an action on $\mathbb{M}=\mathcal{E}^{1, n} / \Gamma$. This implies that, for every $\left(\mathbf{Z}, \tau^{\prime}\right) \in \Gamma$ and every $(\mathbf{X}, \tau) \in \widehat{\mathrm{O}}_{+}^{\uparrow}(2, n+1)$, we have

$$
\mathrm{p}_{\mathbb{M}} \circ\left(\widehat{\mathrm{L}}_{(\mathbf{X}, \tau)} \circ \widehat{\mathrm{L}}_{\left(\mathbf{Z}, \tau^{\prime}\right)} \circ \widehat{\mathrm{L}}_{(\mathbf{X}, \tau)^{-1}}\right)=\mathrm{L}_{(\mathbf{X}, \tau)} \circ \mathrm{p}_{\mathbb{M}} \circ \widehat{\mathrm{L}}_{(\mathbf{X}, \tau)^{-1}}=\mathrm{p}_{\mathbb{M}} .
$$

Then, for every $\left(\mathbf{Z}, \tau^{\prime}\right) \in \Gamma$, the image of the map

$$
f_{\left(\mathbf{Z}, \tau^{\prime}\right)}: \widehat{\mathrm{O}}_{+}^{\uparrow}(2, n+1) \ni(\mathbf{X}, \tau) \longmapsto(\mathbf{X}, \tau) \star\left(\mathbf{Z}, \tau^{\prime}\right) \star(\mathbf{X}, \tau)^{-1} \in \widehat{\mathrm{O}}_{+}^{\uparrow}(2, n+1)
$$

is contained in $\Gamma$. Since $\Gamma$ is discrete and $\widehat{\mathrm{O}}_{+}^{\uparrow}(2, n+1)$ is connected, $f_{\left(\mathbf{Z}, \tau^{\prime}\right)}$ is constant, equal to $\left(\mathbf{Z}, \tau^{\prime}\right)$. Therefore, $\Gamma$ is a subgroup of the center $\widehat{Z}(2, n+1)$ of $\widehat{\mathrm{O}}_{+}^{\uparrow}(2, n+1)$. According to Theorem $\mathrm{A}$ we have the following.

- If $n$ is even, $\Gamma=\{(I, 2 \pi m k) \mid m \in \mathbb{Z}\}$, for some positive integer $k$. Hence, $\mathbb{M}=\mathcal{E}^{1, n} / \Gamma=\mathcal{E}_{\mathrm{I}, k}^{1, n}$.

- If $n$ is odd, then either $\Gamma=\{(I, 2 \pi m k) \mid m \in \mathbb{Z}\}$, for some positive integer $k$, or $\Gamma=\left\{\left((-1)^{m} I, \pi m(1+2 k)\right) \mid m \in \mathbb{Z}\right\}$, for some positive integer $k$. In the first case, $\mathbb{M}=\mathcal{E}^{1, n} / \Gamma=\mathcal{E}_{\mathrm{I}, k}^{1, n}$; in the second case, $\mathbb{M}=\mathcal{E}^{1, n} / \Gamma=\mathcal{E}_{\mathrm{II}, k}^{1, n}$. 
This concludes the proof of Theorem C.

\section{REFERENCES}

[1] D. Alekseevsky, Groups of conformal transformations of Riemannian spaces, Math. Sb. (N.S.) 89(131) (1972), 280-296; translation in Math. USSR-Sb. 18 (1972), no. 2, 285-301.

[2] D. Alekseevsky, $\mathrm{S}^{n}$ and $\mathrm{E}^{n}$ are the only Riemannian spaces with essential conformal transformations, Uspekhi Math. Nauk. 28 (1973), no. 5, 289-290.

[3] D. Alekseevsky, Lorentzian manifolds with transitive conformal group, Note Mat. 37 (2017), suppl. 1, 35-47; e-print: arXiv:1611.03447 [math.DG].

[4] V. Bargmann, Irreducible unitary representations of the Lorentz group, Ann. of Math. (2) 48 (1947), 568-640.

[5] V. Bargmann, Group representations in Hilbert spaces of analytic funcions, in Analytical Methods in Mathematical Physics, 27-63, P. Gilbert and R. G. Newon, Eds. Gordon and Breach, New York, 1970.

[6] T. Barbot, V. Charette, T. Drumm, W. M. Goldman, and K. Melnick, A primier on the (2+1)-Einstein universe, in Recent developments in pseudo-Riemannian geometry, 179-229, ESI Lect. Math. Phys., Eur. Math. Soc., Zürich, 2008; arXiv:0706.3055[math.DG].

[7] A. Borel, Les espaces hermitiens symétriques, Séminaire Bourbaki, Tome 2 (1951-1954), Exposé no. 62, 121-132.

[8] G. E. Bredon, Introduction to Compact Transformation Groups, Pure and Applied Mathematics, 46, Academic Press, New York-London, 1972.

[9] M. Cahen and Y. Kerbrat, Domaines symétriques des quadriques projectives, J. Math. Pures Appl. (9) 62 (1983), no. 3, 327-348.

[10] E. Cartan, Sur les domaines bornés homogènes de l'espace de $n$ variables complexes, $A b h$. Math. Sem. Univ. Hamburg 11 (1936), no. 1, 116-162.

[11] E. Cartan, Les espaces à connexion conforme, Ann. Soc. Pol. Math. 2 (1923), 171-221.

[12] T. E. CECIL, Lie sphere geometry: with applications to submanifolds, Springer-Verlag, New York, 1992.

[13] P. T. Chruściel, G. J. Galloway, and D. Pollack, Mathematical general relativity: a sampler, Bull. Amer. Math. Soc. (N.S.) 47 (2010), 567-638.

[14] A. Dzhalilov, E. Musso, and L. Nicolodi, Conformal geometry of timelike curves in the (1+2)-Einstein universe, Nonlinear Anal., 143 (2016), 224-255.

[15] A. Einstein, Kosmologische Betrachtungen zur allgemeinen Relativitätstheorie, Sitzungsberichte der Königlich Preussischen Akademie der Wissenschaften, 142-152, Berlin, 1917.

[16] S. W. Hawking and G. F. R. Ellis, The Large Scale Structure of Space-time, Cambridge Monographs on Mathematical Physics, no. 1, Cambridge University Press, London-New York, 1973.

[17] S. Helgason, Differential Geometry, Lie Groups, and Symmetric Spaces, Academic Press, New York, 1978.

[18] L.-K. HuA, On the theory of Fuchsian functions of several variables, Ann. of Math. (2) 47 (1946), no. 2, 167-191.

[19] L.-K. HuA, Harmonic Analysis of Functions of Several Complex Variables in the Classical Domains, Translations of Mathematical Monographs, 6, Amer. Math. Soc., Providence, RI, 1963.

[20] S. Kobayashi And K. Nomizu, Foundations of Differential Geometry, Vol. I, Interscience Publishers, John Wiley \& Sons, New York-London, 1963.

[21] S. Kobayashi, Transformation Groups in Differential Geometry, Ergebnisse der Mathematik und ihrer Grenzgebiete, 70, Springer-Verlag, New York-Heidelberg, 1972.

[22] R. S. Kulkarni and U. Pinkall (Eds.), Conformal Geometry, Aspects of Mathematics, E12, Friedr. Vieweg \& Sohn, Braunschweig, 1988.

[23] J. LeLOnG-FerRand, Transformations conformes et quasi-conformes des variétés riemanniennes compactes (démonstration de la conjecture de A. Lichnerowicz), Acad. Roy. Belg. Cl. Sci. Mém. Coll. 39 (1971), no. 5, 44 pages.

[24] K. YAno, Integral Formulas in Riemannian Geometry, Pure and Applied Mathematics, 1, Marcel Dekker, Inc., New York, 1970.

[25] H. B. Lawson and M. L. Michelsohn, Spin Geometry, Princeton University Press, Princeton, NJ, 1989 
[26] M. Овата, The conjectures on conformal transformations of Riemannian manifolds, J. Differential Geom. 6 (1971), 247-258.

[27] B. O'NeIll, Semi-Riemannian Geometry with Applications to Relativity, Pure and Applied Mathematics, 103, Academic Press, Inc., New York, 1983.

[28] M. N. Podoksenov, Conformally homogeneous Lorentz manifolds, Sibirsk. Mat. Zh. 33 (1992), no. 6, 154-161; translation in Siberian Math. J. 33 (1992), no. 6, 1087-1093.

[29] J. Rawnsley, On the universal covering of the real symplectic group, J. Geom. Phys. 62 (2012), no. 10, 2044-2058.

[30] P. Robinson and J. Rawnsley, The Metaplectic Representation, $\mathrm{Mp}^{c}$ Structures and Geometric Quantization, Memoirs of the AMS, Vol. 410, American Mathematical Society, Providence, RI, 1989.

[31] I. Satake, Algebraic Structures of Symmetric Domains, Kanô Memorial Lectures, Vol. 4, Iwanami Shoten, Tokyo, 1980.

[32] N. Steenrod, Topology of Fibre Bundles, Princeton University Press, Princeton, NJ, 1951.

(O. Eshkobilov) Dipartimento di Matematica "Giuseppe Peano", Università di Torino, Via Carlo Alberto 10, I-10123 Torino, Italy

E-mail address: olimjon.eshkobilov@edu.unito.it, olimjon.eshkobilov@polito.it

(E. Musso) Dipartimento di Scienze Matematiche, Politecnico di Torino, Corso Duca Degli Abruzzi 24, I-10129 Torino, Italy

E-mail address: emilio.musso@polito.it

(L. Nicolodi) Dipartimento di Scienze Matematiche, Fisiche e Informatiche, Università di Parma, Parco Area delle Scienze 53/A, I-43124 Parma, Italy

E-mail address: lorenzo.nicolodi@unipr.it 